\title{
Anomalous Scaling from Controlled Closure in a Shell Model of Turbulence
}

\author{
Victor S. L'vov, Daniela Pierotti, Anna Pomyalov and Itamar Procaccia \\ Department of Chemical Physics, The Weizmann Institute of Science, Rehovot 76100, Israel
}

\begin{abstract}
We present a model of hydrodynamic turbulence for which the program of computing the scaling exponents from first principles can be developed in a controlled fashion. The model consists of $N$ suitably coupled copies of the "Sabra" shell model of turbulence. The couplings are chosen to include two components: random and deterministic, with a relative importance that is characterized by a parameter called $\epsilon$. It is demonstrated, using numerical simulations of up to 25 copies and 28 shells that in the $N \rightarrow \infty$ limit but for $0<\epsilon \leq 1$ this model exhibits correlation functions whose scaling exponents are anomalous. The theoretical calculation of the scaling exponents follows verbatim the closure procedure suggested recently for the Navier-Stokes problem, with the additional advantage that in the $N \rightarrow \infty$ limit the parameter $\epsilon$ can be used to regularize the closure procedure. The main result of this paper is a finite and closed set of scale-invariant equations for the 2nd and 3rd order statistical objects of the theory. This set of equations takes into account terms up to order $\epsilon^{4}$ and neglects terms of order $\epsilon^{6}$. Preliminary analysis of this set of equations indicates a K41 normal scaling at $\epsilon=0$, with a birth of anomalous exponents at larger values of $\epsilon$, in agreement with the numerical simulations.
\end{abstract}

It is almost impossible to write a paper in the area of fluid turbulence without drawing heavily on one or more papers of Bob Kraichnan, who usually introduced the issue and wrote one or more fundamental papers towards its resolution. The present contribution is not an exception. It uses freely the model that Bob has introduced in [1]. Bob, we are happy to dedicate this paper to you at the occasion of your 70th birthday. The interaction, intellectual stimulation and friendship that you gave us over the last decade are unforgettable, and we hope and trust that they will continue for years to come.

\section{INTRODUCTION}

In this paper we present a calculation scheme aimed at evaluating the scaling exponents that characterize correlation functions of turbulent fields. For the sake of clarity we consider shell models of turbulence rather than analyze the Navier-Stokes equations. However we stress from the start that in principle all the steps provided here can be repeated in the context of Navier-Stokes turbulence.

Shell models as well as Navier-Stokes turbulence pose an infinite hierarchy of dynamical equations for the norder correlation functions. This hierarchy is linear in the correlation functions, and in the limit of infinite Reynolds number is also homogeneous. It was recently discovered [2,3] that this hierarchy obeys a rescaling symmetry which stems from the rescaling symmetry of the Euler equation [4]. This rescaling symmetry foliates the space of solutions into slices of different scaling exponents $h$ of the velocity; these are referred to as $h$-slices. On each $h$-slice one finds "normal scaling" with the given value of $h$. The full solution is a linear combination of all the solutions on the $h$-slices with non-universal weights which are determined by the forcing on the integral scale of turbulence. Different orders of the correlation functions are dominated by different $h$-slices, and accordingly the full solution has anomalous scaling. The anomalous ex- ponents are expected to be universal.

In trying to evaluate the scaling exponents appearing in this way from first principles, we proposed [2, 3, 5] to truncate the hierarchy of equations, preserving the fundamental rescaling symmetry that gives rise to anomalous scaling. Truncation is problematic; in turbulence there is no natural small parameter, and therefore any closure of an infinite hierarchy is uncontrolled. It is therefore worthwhile to introduce a 1-parameter family of models, characterized by a parameter $\epsilon \in[0,1]$, which shows normal scaling when $\epsilon=0$ and recovers the anomalous scaling of the original model when $\epsilon=1$.

Following an idea in [1], we will construct such a family, and show that the transition from normal to anomalous behavior occurs at some finite value of $\epsilon>0$. We will use $\epsilon$ as a small parameter to regularize the closure procedure; we will show that our closed equations are valid to $O\left(\epsilon^{4}\right)$, whereas the neglected terms are of $O\left(\epsilon^{6}\right)$. We can improve the closure scheme systematically by including terms of $O\left(\epsilon^{6}\right)$, neglecting terms of $O\left(\epsilon^{8}\right)$, etc.

A way to achieve this small parameter is to couple $N$ copies of the same turbulent system, be it a shell model or the Navier-Stokes equations, and choose the coupling to have both a deterministic and a random contributions, with relative amplitudes $\epsilon$ and $\sqrt{1-\epsilon^{2}}$. For $\epsilon=1$ we loose the coupling between the copies, and recover the 
initial anomalous problem for any value of $N$. For $\epsilon=0$ we will show that that in the limit $N \rightarrow \infty$ we get normal scaling. Thus for some value of $\epsilon>0$ and for large enough $N$ we expect to see the birth of anomalous scaling, hopefully in a perturbative fashion. The existence of this transition is the main discovery of this paper, and we study it analytically using the $\epsilon$-controlled closure procedure, and by direct simulations of the $N$-copied model.

In Section 2 we review briefly the "Sabra" shell model of turbulence [6], and introduce the copy space with the appropriate coupling. We study the resulting model numerically in Sect. 3. In Section 4 we start the construction of the theory. First we present the statistical objects and derive the hierarchy of evolution equations that they satisfy, exposing in particular their scale invariance. In Section 5 we discuss the closure which preserves the scaleinvariance, demonstrating how the smallness of $\epsilon$ is used to control consecutive closure steps. Sections 6 and 7 are devoted to analysis of the resulting $\epsilon$-controlled closure, and the results are compared in Sect. 8 with the numerical findings of Sect. 3. The paper is on the whole rather technical, and for the sake of the casual reader we summarize in some detail the points of principle in the conclusion section which is numbered 9 .

\section{THE $(N, \epsilon)$-SABRA MODEL WITH RANDOM COUPLINGS}

\section{A. The Sabra model}

The starting point is the Sabra shell model as introduced in [6]:

$$
\begin{aligned}
& \frac{d u_{n}(t)}{d t}=i\left[a k_{n+1} u_{n+1}^{*} u_{n+2}+b k_{n} u_{n-1}^{*} u_{n+1}\right. \\
& \left.-c k_{n-1} u_{n-2} u_{n-1}\right]-\nu k_{n}^{2} u_{n}+f_{n}(t) \text {. }
\end{aligned}
$$

Here $u_{n}$ refers to the amplitude associated with "wavevector" $k_{n}$, where the spacing in this reduced model is determined by $k_{n} \equiv k_{0} \lambda^{n} ; \lambda$ is the spacing parameter, $\nu$ the "viscosity", $f_{n}(t)$ a random Gaussian force which is operating on the lowest shells. The parameters $a, b$ and $c$ are restricted by the requirement

$$
a+b+c=0,
$$

which guarantees the conservation of the "energy"

$$
E=\sum_{n=0}^{N}\left|u_{n}(t)\right|^{2},
$$

in the inviscid, unforced limit.

The equations of the Sabra model are invariant under the phase transformation

$$
u_{n} \rightarrow u_{n} \exp \left(i \theta_{n}\right)
$$

where the phases $\theta_{n}$ are restricted by the set of equations

$$
\theta_{n-1}+\theta_{n}=\theta_{n+1}
$$

Choosing $\theta_{1}$ and $\theta_{2}$ arbitrarily, $\theta_{n}$ is determined for all $n>2$. Evidently, the physical results of the model must be independent of the choice of the phases $\theta_{1}$ and $\theta_{2}$. In particular, the only non-zero correlation functions are those which are independent of the phases $\theta_{n}$. The nonvanishing $2 \mathrm{nd}$ and $3 \mathrm{rd}$ order correlators are

$$
\begin{aligned}
& S_{2}\left(k_{n}\right)=\left\langle\left|u_{n}\right|^{2}\right\rangle, \\
& S_{3}\left(k_{n}\right)=\operatorname{Im}\left\langle u_{n-1} u_{n} u_{n+1}^{*}\right\rangle .
\end{aligned}
$$

Note that Eq. (1) respects additional phase symmetry

$$
u_{n} \rightarrow-u_{n}^{*}
$$

One of the consequences of this symmetry is that

$$
\operatorname{Re}\left\langle u_{n-1} u_{n} u_{n+1}^{*}\right\rangle=0
$$

explaining why we only need to consider the imaginary part in Eq. (7). The symmetries of this model were selected explicitly in [6] to give rise to a small number of nonzero correlation functions, with the aim of simplifying the calculations presented in the later sections of this paper.

\section{B. $(N, \epsilon)$-generalization of the Sabra model}

The standard available procedures to generalize dynamical systems to $N$ copies involve real variables [7]. In shell models in general, and in the Sabra model in particular, the amplitudes $u_{n}$ are complex. Therefore we rewrite Eq. (1), following [8], in terms of the real and imaginary parts $u_{n}^{\prime} \equiv \operatorname{Re}\left\{u_{n}\right\}$ and $u_{n}^{\prime \prime} \equiv \operatorname{Im}\left\{u_{n}\right\}$. Doing this we guarantee, after the generalization to $N$ copies, that the $N \rightarrow 1$ limit coincides with the original model. The equations are:

$$
\begin{aligned}
& \frac{d u_{n}^{\prime}(t)}{d t}=\left[\gamma_{a, n+1}\left(-u_{n+1}^{\prime} u_{n+2}^{\prime \prime}+u_{n+1}^{\prime \prime} u_{n+2}^{\prime}\right)\right. \\
& \quad+\gamma_{b, n}\left(-u_{n-1}^{\prime} u_{n+1}^{\prime \prime}+u_{n-1}^{\prime \prime} u_{n+1}^{\prime}\right)+\gamma_{c, n-1}\left(u_{n-2}^{\prime} u_{n-1}^{\prime \prime}\right. \\
& \left.\left.\quad+u_{n-2}^{\prime \prime} u_{n-1}^{\prime}\right)\right]-\nu k_{n}^{2} u_{n}^{\prime}+f_{n}^{\prime}(t) \\
& \frac{d u_{n}^{\prime \prime}(t)}{d t}=\left[\gamma_{a, n+1}\left(u_{n+1}^{\prime} u_{n+2}^{\prime}+u_{n+1}^{\prime \prime} u_{n+2}^{\prime \prime}\right)\right. \\
& \quad+\gamma_{b, n}\left(u_{n-1}^{\prime} u_{n+1}^{\prime}+u_{n-1}^{\prime \prime} u_{n+1}^{\prime \prime}\right)+\gamma_{c, n-1}\left(-u_{n-2}^{\prime} u_{n-1}^{\prime}\right. \\
& \left.\left.\quad+u_{n-2}^{\prime \prime} u_{n-1}^{\prime \prime}\right)\right]-\nu k_{n}^{2} u_{n}^{\prime \prime}+f_{n}^{\prime \prime}(t)
\end{aligned}
$$

where

$$
\gamma_{a, n} \equiv a k_{n}, \quad \gamma_{b, n} \equiv b k_{n}, \quad \gamma_{c, n} \equiv c k_{n}
$$


These equations can be written more compactly using the following matrices:

$$
\begin{array}{rlrl}
\boldsymbol{A}^{+1} & \equiv\left(\begin{array}{cc}
1 & 0 \\
0 & 1
\end{array}\right), & \boldsymbol{A}^{-1} \equiv\left(\begin{array}{cc}
0 & -1 \\
1 & 0
\end{array}\right), \\
\boldsymbol{C}^{+1} \equiv\left(\begin{array}{cc}
-1 & 0 \\
0 & 1
\end{array}\right), & \boldsymbol{C}^{-1} \equiv\left(\begin{array}{ll}
0 & 1 \\
1 & 0
\end{array}\right),
\end{array}
$$

which also may be written as $A_{\sigma^{\prime} \sigma^{\prime \prime}}^{\sigma}$ and $C_{\sigma^{\prime} \sigma^{\prime \prime}}^{\sigma}$ with $\sigma, \sigma^{\prime}, \sigma^{\prime \prime}= \pm 1$. The first subscript $\sigma^{\prime}$ denotes line $(+1$ corresponds to the upper line), second subscript $\sigma^{\prime \prime}$ denotes column $(+1$ corresponds to the left one). Clearly

$$
A_{\sigma^{\prime} \sigma^{\prime \prime}}^{\sigma}=A_{\sigma \sigma^{\prime \prime}}^{\sigma^{\prime}}, \quad A_{\sigma^{\prime} \sigma^{\prime \prime}}^{\sigma}=C_{\sigma^{\prime \prime} \sigma}^{\sigma^{\prime}} .
$$

Denoting $u_{n,-1} \equiv u_{n}^{\prime}, u_{n,+1} \equiv u_{n}^{\prime \prime}$ we rewrite Eqs. (10, 11) as

$$
\begin{aligned}
& \frac{d u_{n, \sigma}}{d t}=\left[A _ { \sigma ^ { \prime } \sigma ^ { \prime \prime } } ^ { \sigma } \left(\gamma_{a, n+1} u_{n+1, \sigma^{\prime}} u_{n+2, \sigma^{\prime \prime}}\right.\right. \\
& \left.\left.\quad+\gamma_{b, n} u_{n-1, \sigma^{\prime}} u_{n+1, \sigma^{\prime \prime}}\right)+C_{\sigma^{\prime} \sigma^{\prime \prime}}^{\sigma} \gamma_{c, n-1} u_{n-2, \sigma^{\prime}} u_{n-1, \sigma^{\prime \prime}}\right] \\
& -\nu k_{n}^{2} u_{n, \sigma}+f_{n, \sigma} .
\end{aligned}
$$

As usual, we adopt the convention that repeated dummy indices (here $\sigma^{\prime}$ and $\sigma^{\prime \prime}$ ) are summed upon.

Next consider $N$ copies of Eq. (15). The copies are indexed by $i, j$ or $\ell$, and these indices take on values $-J, \ldots,+J, 2 J+1=N, N$ odd. The $i$ th copy is denoted as $u_{n, \sigma}^{[i]}$. Let $D^{[i j \ell]}$ be the coupling between copies, which will be chosen later. Equations (15) for a collection of copies are written as

$$
\begin{aligned}
& \frac{d u_{n, \sigma}^{[i]}}{d t}=\sum_{j \ell} D^{[i j \ell]}\left[A _ { \sigma ^ { \prime } \sigma ^ { \prime \prime } } ^ { \sigma } \left(\gamma_{a, n+1} u_{n+1, \sigma^{\prime}}^{[j]} u_{n+2, \sigma^{\prime \prime}}^{[\ell]}\right.\right. \\
& \left.\left.\quad+\gamma_{b, n} u_{n-1, \sigma^{\prime}}^{[j]} u_{n+1, \sigma^{\prime \prime}}^{[\ell]}\right)+C_{\sigma^{\prime} \sigma^{\prime \prime}}^{\sigma} \gamma_{c, n-1} u_{n-2, \sigma^{\prime}}^{[j]} u_{n-1, \sigma^{\prime \prime}}^{[\ell]}\right] \\
& \quad-\nu k_{n}^{2} u_{n, \sigma}^{[i]}+f_{n, \sigma}^{[i]} .
\end{aligned}
$$

To proceed we note that the index $\ell$ is defined modulo $N$, and introduce a Fourier transform in the "copy" space:

$$
u_{n, \sigma}^{\alpha}=\frac{1}{\sqrt{N}} \sum_{\ell=-J}^{J} u_{n, \sigma}^{[\ell]} \exp \left(\frac{2 i \pi \alpha \ell}{N}\right) .
$$

Note that the index $\alpha$ is also defined modulo $N=2 J+1$. It is convenient to consider values $\alpha$ within "the first Brillouin zone", i.e from $-J$ to $J$. We will refer to it as the $\alpha$-momentum. Since $u_{n, \sigma}^{[i]}$ is real,

$$
u_{n, \sigma}^{-\alpha}=\left(u_{n, \sigma}^{\alpha}\right)^{*} \equiv u_{n, \sigma}^{\alpha *} .
$$

In " $\alpha$-Fourier space" Eqs. (16) read

$$
\begin{aligned}
& \frac{d u_{n, \sigma}^{\alpha}}{d t}=\sum_{\beta, \gamma} \Phi^{\alpha, \beta, \gamma}\left[\Delta_{\alpha, \beta+\gamma}+\Delta_{\alpha+N, \beta+\gamma}+\Delta_{\alpha, \beta+\gamma+N}\right] \\
& \times\left\{A_{\sigma^{\prime} \sigma^{\prime \prime}}^{\sigma}\left[\gamma_{a, n+1} u_{n+1, \sigma^{\prime}}^{\beta} u_{n+2, \sigma^{\prime \prime}}^{\gamma}+\gamma_{b, n} u_{n-1, \sigma^{\prime}}^{\beta} u_{n+1, \sigma^{\prime \prime}}^{\gamma}\right]\right. \\
& \left.+C_{\sigma^{\prime} \sigma^{\prime \prime}}^{\sigma} \gamma_{c, n-1} u_{n-2, \sigma^{\prime}}^{\beta} u_{n-1, \sigma^{\prime \prime}}^{\gamma}\right\}-\nu k_{n}^{2} u_{n, \sigma}^{\alpha}+f_{n, \sigma}^{\alpha} .
\end{aligned}
$$

where $\Delta_{\alpha, \beta}$ is the Kronecker symbol: $\Delta_{\alpha, \alpha}=1$ and $\Delta_{\alpha, \beta}=0$ for $\alpha \neq \beta$. Observe that we use Greek indices for denoting component in $\alpha$-Fourier space, and Latin indices for copies in the copy space. As a consequence of the discrete translation symmetry of the copy index $[i]$ Eqs. (19) conserve $\alpha$-momentum modulo $N$ at the nonlinear vertex, as one can see explicitly in the above equation. The coupling amplitudes $\Phi^{\alpha, \beta, \gamma}$ in these equations are the Fourier transforms of the coupling amplitudes $D^{[i j \ell]}$. Our choice of these amplitudes will be presented in the next subsection.

\section{Choice of coupling}

We choose the coupling amplitudes according to

$$
\Phi^{\alpha, \beta, \gamma}=\frac{1}{\sqrt{N}}\left[\epsilon+\sqrt{1-\epsilon^{2}} \Psi^{\alpha, \beta, \gamma}\right]
$$

where $\Psi^{\alpha, \beta, \gamma}$ are quenched random phases chosen with uniform distribution of the phase, independently distributed with zero average. The meaning of quenched randomness in the context of a direct numerical simulation is that we run Eq. (19) with a given random choice of $\Phi$, obtain results averaged over the randomness of the forcing $f$, and then rerun with a fresh random choice of $\Phi$. Only at the end we average the statistical objects over the runs. It is believed [9], and checked numerically in this work, that when the number $N \rightarrow \infty$ the statistical functions are self averaging, and the last average is unneeded.

The couplings satisfy the following symmetries:

$$
\begin{aligned}
& \Psi^{\alpha, \beta, \gamma}=\Psi^{\alpha, \gamma, \beta}, \quad\left(\Psi^{\alpha, \beta, \gamma}\right)^{*}=\Psi^{-\alpha,-\beta,-\gamma}, \\
& \Psi^{\alpha, \beta, \gamma}=\Psi^{-\gamma, \beta,-\alpha} .
\end{aligned}
$$

The first of these conditions stems from the identity of copies, leading to the invariance of the equations of motion to an interchange of copies in the nonlinear term. The second one is the reality conditions, the third imposes energy conservation in the inviscid, unforced limit. The requirement

$$
\Psi^{\alpha, \beta, \gamma}=1 \quad \text { if } \quad \alpha \beta \gamma=0
$$

guarantees that for $N=1$ we recapture the original model at any $\epsilon$. Note that for $\epsilon=0$ we have the so called "Random Coupling Model" proposed in the context of the Navier-Stokes statistics by Kraichnan in [7]. It was understood [7.9] that in the limit $N \rightarrow \infty$ the direct interaction approximation (DIA) becomes exact. After 
proper elimination of the sweeping effect (in the framework on the Lagrangian-history DIA [10] or by means of the Belinicher-L'vov approach [11]) the Kolmogorov 1941 (K41) scaling appear as an exact solution of the Random Coupling Model. The same is true for the shell models [8,9] in which the sweeping effect is absent by construction. For $\epsilon=1$ the coupling coefficients in the $\alpha$-Fourier space (20) are index-independent. This corresponds to uncoupling the equations (16) in the copy space, because in this case $D^{[i j \ell]}=\delta_{i, j} \delta_{i, \ell}$. Thus for $\epsilon=1$ we recover the original Sabra model with anomalous scaling [6]. We see that with the choice of the couplings (20) the model interpolates continuously between the "Random Coupling Model" for $\epsilon=0$ whose scaling is normal K41 (at $N \rightarrow \infty$ ) and the Sabra model with anomalous scaling for $\epsilon=1$. A model of this type was proposed in the context of the Navier-Stokes statistics by Kraichnan in [1]. The consequences for the perturbative theory in the large $N$ and small $\epsilon$ limits were considered by Eyink [9].

\section{NUMERICAL INVESTIGATIONS}

We are interested in the $N \rightarrow \infty$ limit of Eqs. (19) as a function of $\epsilon$. As we discussed above, for $\epsilon=0$ we have the random coupling model with normal K41 scaling in the limit $N \rightarrow \infty$, while for $\epsilon=1$ the copies become uncoupled and with a proper definition of the structure functions all the scaling exponents $\zeta_{n}$ are the same as the scaling exponent of the original Sabra model. We thus expect that for intermediate values of $\epsilon$ we may find values of $\zeta_{n}$ that interpolate between K41 and the Sabra value.

This is actually what was found. We measured the scaling exponent $\zeta_{2}$ of the second order structure function $S_{2}\left(k_{n}\right) \equiv F_{2}\left(k_{n} ; 0\right), F_{2}\left(k_{n} ; t\right)$ being defined by Eq. (33). The exponent $\zeta_{2}$ have been calculated either with a linear fit in the two decade inertial range, from $n=4$ to $n=19$ (see Fig. 11) or by using the fitting procedure on the all structure function introduced in [6]. The results are the same although there is a difference in the estimate of the error bars. The error bars in Fig. 2 are the ones obtained for the linear fit. In Fig. 2 one can see the plot of the value of the anomalous corrections to Kolmogorov scaling, $\delta \zeta_{2}=\zeta_{2}-2 / 3$, as function of $1 / N$ for $\epsilon=0.8$ together with the same curve for $\epsilon=0$ and for $\epsilon=1$ for $N$ ranging from 5 to 25 .

The equations of motion (19) with $a=1, b=c=-0.5$, were integrated with the slaved Adams-Bashforth algorithm, viscosity $\nu=4 \times 10^{-9}$, a time-step $\Delta t=10^{-5}$. The forcing was subjected on the first two shells, chosen random Gaussian with zero average and with variances such that $\sigma_{2} / \sigma_{1}=0.7$ (in order to minimize the input of helicity [6]). Averages were taken for a time equal to

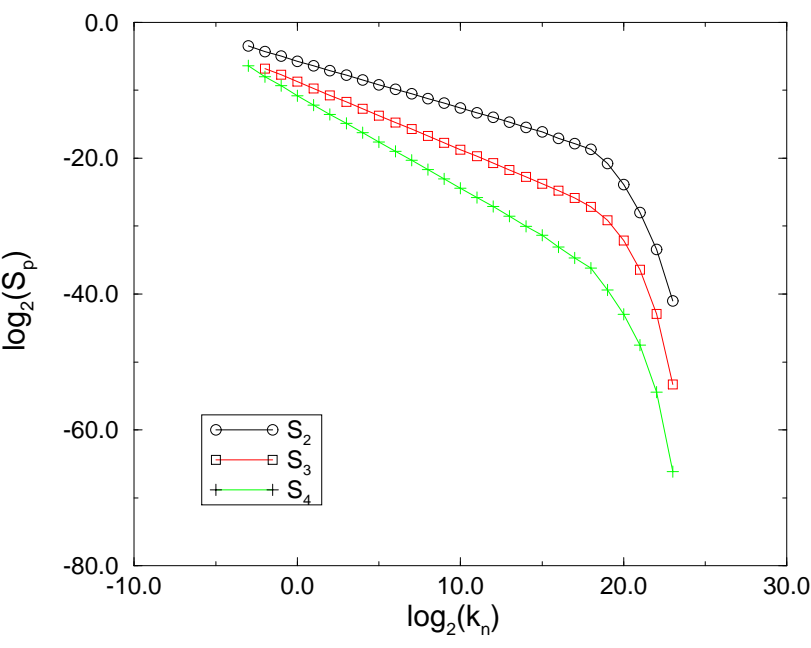

FIG. 1. Structure functions $\log _{2}\left(S_{p}\left(k_{n}\right)\right)$ vs $k_{n}$ in log-log plot for $\mathrm{p}=2,3,4, \epsilon=0.8$ and $N=25$.

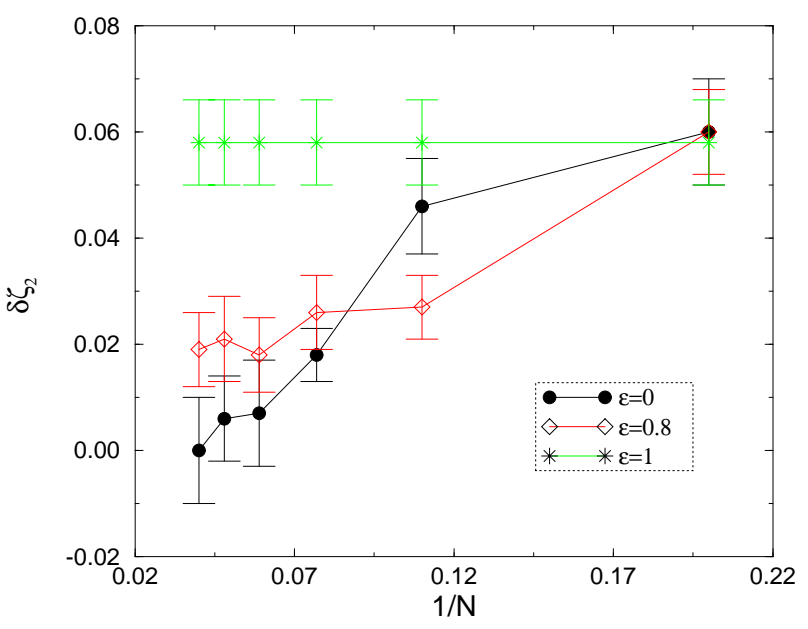

FIG. 2. $\delta \zeta_{2}=\zeta_{2}-2 / 3$ vs $1 / N$ for $\epsilon=0.8$ (diamonds) and $\epsilon=0$ (circles) for $N$ from 5 to 25 .

250 eddy turnover times for the case $N=1$. The averaging times were decreased when the number of copies increased, taking into account the faster convergence times in these cases.

The random couplings were chosen with a zero-mean, uniform probability for the random phase at the beginning of each simulations. Rigorously one should have taken averages over different runs with different couplings but we checked that self-averaging is already valid for $N=5$, at least for $\epsilon=0.8$ (very small random component in the couplings) and within our numerical precision. For $\epsilon=0$ self-averaging occurs for large numbers of copies.

In Fig. 2 it is clear then, while in the case for $\epsilon=0$ the corrections to Kolmogorov scaling goes to zero, as 
was already checked for the equivalent spherical model [8], for $\epsilon=0.8$ and for $\epsilon=1$ the corrections converge to a finite value which increases with $\epsilon$.

\section{THE EQUATIONS OF MOTION OF THE STATISTICAL OBJECTS}

\section{A. Multi-time correlation functions: definitions and symmetries}

The nonzero 2nd and 3rd order multi-time correlation functions of the collective variables are defined as

$$
\begin{aligned}
& F_{2 ; \sigma, \sigma^{\prime}}^{\alpha}\left(k_{n} ; t-t^{\prime}\right) \Delta_{\alpha, \alpha^{\prime}} \equiv\left\langle u_{n, \sigma}^{\alpha}(t) u_{n, \sigma^{\prime}}^{\alpha^{\prime} *}\left(t^{\prime}\right)\right\rangle \\
& F_{3 ; \alpha^{\prime}, \alpha^{\prime \prime}, \sigma^{\prime \prime}}^{\alpha \prime}\left(k_{n} ; t, t^{\prime}, t^{\prime \prime}\right)\left[\Delta_{\alpha+\alpha^{\prime}, \alpha^{\prime \prime}}+\Delta_{\alpha+\alpha^{\prime}, \alpha^{\prime \prime}+N}\right. \\
& \left.\quad+\Delta_{\alpha+\alpha^{\prime}+N, \alpha^{\prime \prime}}\right] \equiv\left\langle u_{n-1, \sigma^{\prime}}^{\alpha}(t) u_{n, \sigma^{\prime}}^{\alpha^{\prime}}\left(t^{\prime}\right) u_{n+1, \sigma^{\prime \prime}}^{\alpha^{\prime \prime} *}\left(t^{\prime \prime}\right)\right\rangle .
\end{aligned}
$$

The conservation of the $\alpha$-momentum which was discussed above causes the 2nd order correlation function to be diagonal in $\alpha, \alpha^{\prime}$, and puts a constraint $\alpha+\alpha^{\prime}=$ $\alpha^{\prime \prime} \bmod N$ on the 3 rd order correlation. In (23) we wrote explicitly that in stationary conditions $F_{2}$ depends on the time difference $t-t^{\prime}$ only; it is clear also that $F_{3}$ depends on two time differences, say $t-t^{\prime}$ and $t-t^{\prime \prime}$. There are a few nonzero 4th order correlation functions, we present here a natural general definition:

$F_{4 ; \sigma_{1}, \sigma_{2}, \sigma_{3}, \sigma_{4}}^{\alpha_{1}, \alpha_{2}, \alpha_{3}, \alpha_{4}}\left(k_{n_{1}}, k_{n_{2}}, k_{n_{3}}, k_{n_{4}} ; t_{1}, t_{2}, t_{3}, t_{4}\right) \Delta_{\alpha_{1}+\alpha_{2}, \alpha_{3}+a_{4}}$ $\equiv\left\langle u_{n_{1}, \sigma_{1}}^{\alpha_{1}}\left(t_{1}\right) u_{n_{2}, \sigma_{2}}^{\alpha_{2}}\left(t_{2}\right) u_{n_{3}, \sigma_{3}}^{\alpha_{3} *}\left(t_{3}\right) u_{n_{4}, \sigma_{4}}^{\alpha_{4} *}\left(t_{4}\right)\right\rangle$.

As we see the correlation functions $\boldsymbol{F}_{n}$ defined by (23)(25) depend on the indices $\alpha$ and $\sigma$. This dependence is determined by symmetry considerations. The original Sabra model (II) is invariant under the transformation (8). This leads to the following symmetry of the $N, \epsilon-$ Sabra model (16):

$$
u_{n, \sigma}^{\alpha} \rightarrow \sigma u_{n,-1}^{\alpha} .
$$

Consequently, all the correlation functions must be invariant under this transformation. For $\boldsymbol{F}_{2}$ it means $F_{2 ; \sigma, \sigma^{\prime}}^{\alpha, \alpha^{\prime}}\left(k_{n} ; t\right)=\sigma \sigma^{\prime} F_{2 ; \sigma, \sigma^{\prime}}^{\alpha, \alpha^{\prime}}\left(k_{n} ; t\right)$ and thus $\sigma \sigma^{\prime}=1$, i.e. diagonality in $\sigma, \sigma^{\prime}$ :

$$
F_{2 ; \sigma, \sigma^{\prime}}^{\alpha}\left(k_{n} ; t\right)=\Delta_{\sigma, \sigma^{\prime}} F_{2, \sigma}^{\alpha}\left(k_{n} ; t\right) .
$$

The nonzero components of the $\boldsymbol{F}_{3}$ tensor are those for which the product $\sigma \sigma^{\prime} \sigma^{\prime \prime}=1$, namely:

$$
F_{3 ; 1,1,1}^{\alpha, \alpha^{\prime} \alpha^{\prime \prime}}, \quad F_{3 ; 1,-1,-1}^{\alpha, \alpha^{\prime} \alpha^{\prime \prime}}, \quad F_{3 ;-1,1,-1}^{\alpha, \alpha^{\prime} \alpha^{\prime \prime}}, \quad F_{3 ;-1,-1,1}^{\alpha, \alpha^{\prime} \alpha^{\prime \prime}} .
$$

The nonzero components of $\boldsymbol{F}_{4}(25)$ are those in which the product $\sigma_{1} \sigma_{2} \sigma_{3} \sigma_{4}=1$. The corresponding list is obvious.
The symmetry of the Sabra model under the phase transformation (4, 50 is more restrictive. For the $(N, \epsilon)$ Sabra model it leads to an invariance under the transformation:

$$
\left[\begin{array}{l}
u_{n,-1}^{\alpha} \\
u_{n,+1}^{\alpha}
\end{array}\right] \rightarrow\left[\begin{array}{cc}
\cos \theta_{n}, & -\sin \theta_{n} \\
\sin \theta_{n}, & \cos \theta_{n}
\end{array}\right]\left[\begin{array}{l}
u_{n,-1}^{\alpha} \\
u_{n,+1}^{\alpha}
\end{array}\right]
$$

when $\theta_{n}+\theta_{n+1}=\theta_{n+2}$. The consequence of this for $\boldsymbol{F}_{2}$ is rather simple: the only invariant combination of the 2nd order correlation function is the trace

$$
F_{2}^{\alpha}\left(k_{n} ; t\right) \equiv \sum_{\sigma= \pm 1} F_{2, \sigma}^{\alpha}\left(k_{n} ; t\right)
$$

Actually we can make an even stronger statement: the action of the random force on the first two shells results in the randomization of the phases $\theta_{1}$ and $\theta_{2}$. Since the other phases are determined, they are also random, and satisfy $\left\langle\cos ^{2} \theta_{n}\right\rangle=\left\langle\sin ^{2} \theta_{n}\right\rangle=1 / 2$. Therefore $F_{2, \sigma}^{\alpha}\left(k_{n} ; t\right)=\frac{1}{2} F_{2}^{\alpha}\left(k_{n} ; t\right)$.

Moreover, the function $F_{2}^{\alpha}\left(k_{n} ; t\right)$ is $\alpha$-independent

$$
F_{2, \sigma}^{\alpha}\left(k_{n} ; t\right)=\frac{1}{2} F_{2}\left(k_{n} ; t\right) .
$$

This is equivalent to the statement that the correlation functions are diagonal in the copy space:

$$
\left\langle u_{n, \sigma}^{[\ell]} u_{n, \sigma}^{\left[\ell^{\prime}\right] *}\right\rangle \propto \Delta_{\ell, \ell^{\prime}},
$$

To see this note that the deterministic part of coupling in Eq. (16) is diagonal in the copy space, i.e. $\propto \epsilon \Delta_{i, j} \Delta_{i, \ell}$. The "inter-copy" part of coupling (which is $\propto \sqrt{1-\epsilon^{2}}$ ) is random (being a Fourier sum of the random phases $\left.\Psi^{\alpha, \beta, \gamma}\right)$. Therefore every realization of $\Psi$ 's implies different phase relationships between the variables $u_{n, \sigma}^{[\ell]}$ with different values of $\ell$. Therefore the only second-order correlation functions that survive the $\Psi$-ensemble averaging are those that are diagonal in $\ell, \ell^{\prime}$.

Finally we conclude that among the group (23) of 2nd order correlation function there exist only one object, $F_{2}\left(k_{n} ; t\right)$, which is invariant under all the symmetry transformations of the model and is independent of the indexes $\alpha$ and $\sigma$. In terms of (23) it may be written as:

$$
F_{2}\left(k_{n} ; t\right)=\frac{1}{N} \sum_{\alpha, \sigma} F_{2 ; \sigma, \sigma}^{\alpha}\left(k_{n} ; t\right) .
$$

In a similar but much more involved manner one may find the unique invariant object among the group (24) of 3rd order correlation functions:

$$
\begin{gathered}
F_{3}\left(k_{n} ; t, t^{\prime}, t^{\prime \prime}\right)=\frac{1}{N} \sum_{a, \beta, \gamma} \sum_{\sigma, \sigma^{\prime} \sigma^{\prime \prime}} \Phi^{\gamma, \beta, \alpha} A_{\sigma^{\prime} \sigma^{\prime \prime}}^{\sigma}\left[\Delta_{\alpha, \beta+\gamma}\right. \\
\left.+\Delta_{\alpha+N, \beta+\gamma}+\Delta_{\alpha, \beta+\gamma+N}\right] F_{3 ; \sigma, \sigma^{\prime}, \sigma^{\prime \prime}}^{\alpha, \beta, \gamma}\left(k_{n} ; t, t^{\prime}, t^{\prime \prime}\right) .
\end{gathered}
$$

The easiest way to derive this expression is to compute a time derivative of the invariant object $F_{2}(33)$ as is done in Appendix A1. The only objects which may appear in the result are invariant combinations of the 3rd 
order correlation functions and this is exactly the combination (34). Computing a time derivative of $F_{3}$ (34) we find the following invariant combination of the 4 th order group (25):

$$
\begin{gathered}
F_{4}\left(k_{n} ; t_{1}, t_{2}, t_{3}, t_{4}\right) \equiv \frac{1}{N^{2}} \sum_{\alpha, \beta} \sum_{\sigma_{1}, \cdots \sigma_{5}} A_{\sigma_{2} \sigma_{3}}^{\sigma_{1}} A_{\sigma_{4} \sigma_{5}}^{\sigma_{1}} \\
\times F_{4 ; \sigma_{2}, \sigma_{3}, \sigma_{4}, \sigma_{1}}\left(k_{n+1}, k_{n}, k_{n+1}, k_{n} ; t_{2}, t_{3}, t_{4}, t_{1}\right) .
\end{gathered}
$$

There are few other invariant combinations of the 4th order group (25), but only the combination (35) appears in the dynamics.

Note that the normalization constants $N^{-1}$ in the definitions (33, 34) and $N^{-2}$ in (35) are chosen such that $F_{2}$, $F_{3}$ and $F_{4}$ are of $O(1)$. Moreover note that although the triple correlators (24) are complex functions the invariant combination $F_{3}(34)$ is real as a consequence of the reality condition for $u^{\alpha}$ and of the sums over $\alpha$ and $\beta$. In addition, all these definitions of correlation functions coincide with the original correlations of the Sabra model when $N=1$.

\section{B. Hierarchical equations}

We now present the hierarchy of evolution equations for correlation functions up to the equation for $F_{3}$, which contains $F_{4}$ on its RHS. We will close the hierarchy by expressing $F_{4}$ in terms of lower order objects, showing that what we neglect is of higher order in $\epsilon$.

With the definitions given above, in the inviscid unforced limit (i.e. in the bulk of the inertial range) the evolution equations derived in Appendix A 1 take the form:

$$
\begin{aligned}
\frac{\partial}{\partial t} F_{2}\left(k_{n}, t\right)= & \gamma_{a, n+1} F_{3}\left(k_{n+1} ; 0, t, t\right)+\gamma_{b, n} F_{3}\left(k_{n} ; t, 0, t\right)+\gamma_{c, n-1} F_{3}\left(k_{n-1} ; t, t, 0\right), \\
\frac{\partial}{\partial t_{1}} F_{3}\left(k_{n}, t_{1}, t_{2}, t_{3}\right)= & \gamma_{a, n} F_{4}\left(k_{n}, k_{n+1}, k_{n}, k_{n+1} ; t_{1}, t_{1}, t_{2}, t_{3}\right) \\
& +\gamma_{b, n-1} F_{4}\left(k_{n-2}, k_{n}, k_{n}, k_{n+1} ; t_{1}, t_{1}, t_{2}, t_{3}\right)+\gamma_{c, n-2} F_{4}\left(k_{n-3}, k_{n-2}, k_{n}, k_{n+1} ; t_{1}, t_{1}, t_{2}, t_{3}\right), \quad(37) \\
\frac{\partial}{\partial t_{2}} F_{3}\left(k_{n}, t_{1}, t_{2}, t_{3}\right)= & \gamma_{a, n+1} F_{4}\left(k_{n-1}, k_{n+1}, k_{n+2}, k_{n+1} ; t_{1}, t_{2}, t_{2}, t_{3}\right) \\
& +\gamma_{b, n} F_{4}\left(k_{n-1}, k_{n-1}, k_{n+1}, k_{n+1} ; t_{1}, t_{2}, t_{2}, t_{3}\right)+\gamma_{c, n-1} F_{4}\left(k_{n-1}, k_{n-2}, k_{n-1}, k_{n+1} ; t_{1}, t_{2}, t_{2}, t_{3}\right), \\
\frac{\partial}{\partial t_{3}} F_{3}\left(k_{n}, t_{1}, t_{2}, t_{3}\right)= & \gamma_{a, n+2} F_{4}\left(k_{n-1}, k_{n}, k_{n+2}, k_{n+3} ; t_{1}, t_{2}, t_{3}, t_{3}\right) \\
& +\gamma_{b, n+1} F_{4}\left(k_{n-1}, k_{n}, k_{n}, k_{n+2} ; t_{1}, t_{2}, t_{3}, t_{3}\right)+\gamma_{c, n} F_{4}\left(k_{n-1}, k_{n}, k_{n}, k_{n-1} ; t_{1}, t_{2}, t_{3}, t_{3}\right),
\end{aligned}
$$

This set of equations exhibits terms on the RHS coming from non-linear contributions only. The linear dissipative terms are negligible in the limit $\nu \rightarrow 0$ : there is nothing in the dissipative terms that compensates the vanishing of the viscosity. In addition, the terms coming from the forcing appear explicitly only in the equations for the correlations calculated in $k_{1}$ and $k_{2}$, the forced shells. They vanish in the inertial range.

The structure of the hierarchical Eqs. (36)-(37) and of various future equations becomes transparent if we adopt a graphical representation. The symbols used in the graphical representation are those introduced in the context of renormalized perturbation theory [2,5], see Fig. . It is important to stress however that the present approach is not perturbative, and does not generate any infinite series of diagrams. Accordingly the present theory does not suffer from the usual perturbative problems of unknown convergence properties of infinite series.

The hierarchical Eqs. (36)-(37) are exhibited in Fig. 4a. The consistent closure procedure (see below) requires the introduction of response functions and their equations of motion. The equations for all the response functions up to 4-point objects are presented analytically in Appendix A3 and graphically in Fig. Ab,c.

\section{Scaling invariance and $h$-slices}

The Sabra and $(N, \epsilon)$-Sabra equations (11) and (16) in the unforced, inviscid limit are invariant under the following 2-parameter rescaling group $\mathcal{R}(h, \rho)$ :

$$
\begin{aligned}
& \mathcal{R}(h, \rho) k_{n}=k_{n} / \rho, \quad \mathcal{R}(h, \rho) t=\rho^{1-h} t, \\
& \mathcal{R}(h, \rho) u_{n}=\rho^{h} u_{n},
\end{aligned}
$$

where $\mathcal{R}$ is the rescaling operator. Correspondingly the hierarchical equations for the correlation functions and the Green's functions display invariance under the same rescaling group $\mathcal{R}(h, \rho)$ when the statistical objects transform as follows:

$$
\begin{aligned}
\mathcal{R}(h, \rho) F_{n} & =\rho^{n h} F_{n}, \quad \mathcal{R}(h, \rho) G_{n, 1}=\rho^{(n-1) h-3} G_{n, 1}, \\
\mathcal{R}(h, \rho) G_{1,2} & =\rho^{-(h+6)} G_{1,2}, \ldots
\end{aligned}
$$




\begin{tabular}{|c|c|}
\hline a. Correlation functions: & c. Vertices: \\
\hline $\mathcal{F}_{2}=m m, \quad \mathcal{F}_{3}=m \Omega_{2}^{\xi^{2}}, \mathcal{F}_{4}=1 \sum^{2} m^{3}, .$. & $\begin{array}{cccc}\gamma & \text { A } & \text { B } & \text { C } \\
-\xi & -< & -< & -<\end{array}$ \\
\hline $\begin{array}{l}\text { b. Greens' functions: } \\
\mathcal{G}_{1,1}^{0}=m-, \quad \mathcal{G}_{1,2}=m^{1} \mathcal{L}_{2}^{2}, \quad \mathcal{G}_{2,1}=m^{1},\end{array}$ & 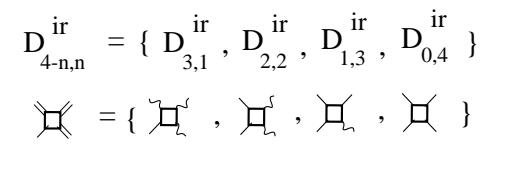 \\
\hline 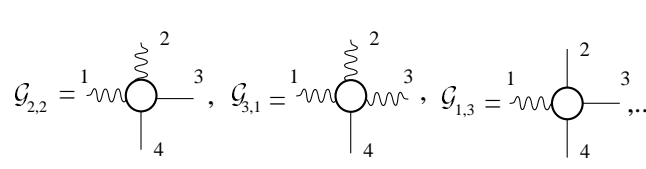 & 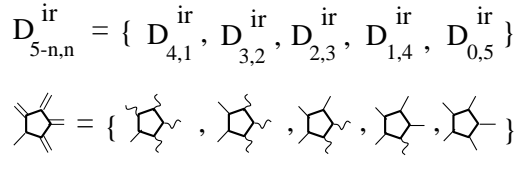 \\
\hline
\end{tabular}

FIG. 3. The diagrammatic notation of the basic objects, Panel a: The correlation functions $\mathcal{F}_{n}$. Panel b: the bare Green's function (thin line) and the dressed Green's functions $\mathcal{G}_{n, m}$. A circle connecting $n$ wavy lines stands for an $n$th order correlation function, and a circle with $n$ wavy lines and $m$ straight lines stands for a Green's function with $n$ velocities and $m$ forcing. Panel c : the 3rd order vertices $\gamma$, and $A, B, C$; irreducible contributions of the 4 th and 5 th order vertices $D_{4-n, n}^{\mathrm{ir}}$ and $D_{5-n, n}$ denoted as empty squares and pentagons. Double line tails stands either for a straight or wavy tail.

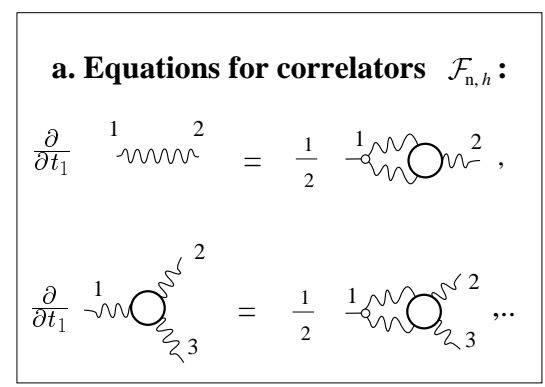

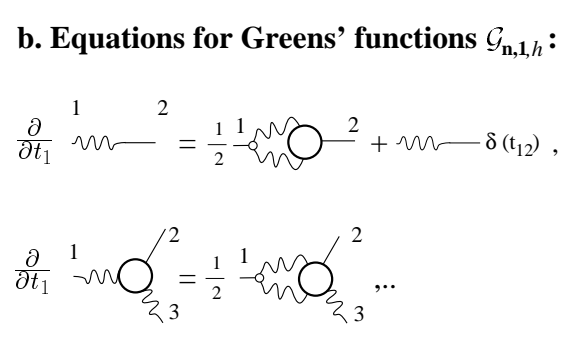

b. Equations for Greens' functions $\mathcal{G}_{\mathbf{n}, 1, h}$ :

c. Equations for Green's functions $\mathcal{G}_{1,2, h}$ :

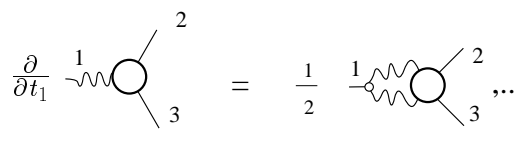

FIG. 4. The symbolic representation of the first evolution equations in the hierarchy for correlation and Green's functions. The graphical notations are presented in Fig. 3

Explicitly, the application of $\mathcal{R}(h, \rho)$ to any statistical object reads:

$$
\begin{aligned}
& \mathcal{R}(h, \rho) F_{n}\left(k_{m}, k_{m^{\prime}}, k_{m^{\prime \prime}}, \cdots ; t, t^{\prime}, t^{\prime \prime}, \cdots\right) \\
\equiv & F_{n}\left(\frac{k_{m}}{\rho}, \frac{k_{m^{\prime}}}{\rho}, \frac{k_{m^{\prime \prime}}}{\rho}, \cdots ; \rho^{1-h} t, \rho^{1-h} t^{\prime}, \rho^{1-h} t^{\prime \prime}, \cdots\right) .
\end{aligned}
$$

The simple fact that the hierarchical equations are linear equations has a crucial consequence for the theory: by direct substitution one can check that the rescaling symmetry is wider then (39) and includes an additional $n$-independent function of $h-\mathcal{Z}(h)$, which at present seems arbitrary:

$$
\begin{aligned}
\mathcal{R}(h, \rho) F_{n} & =\rho^{n h+\mathcal{Z}(h)} F_{n}, \\
\mathcal{R}(h, \rho) G_{n, 1} & =\rho^{(n-1) h-3+\mathcal{Z}(h)} G_{n, 1}, \\
\mathcal{R}(h, \rho) G_{1,2} & =\rho^{-(h+6)+\mathcal{Z}(h)} G_{1,2}, \ldots
\end{aligned}
$$

Note that in principle the rescaling group for the hierarchies of the correlation and Green's function could be defined using two different functions $\mathcal{Z}_{1}(h)$ and $\mathcal{Z}_{2}(h)$. However, the existence of an inhomogeneous term proportional to the bare Green's function (first line in Fig.(4) results in the identify of $\mathcal{Z}(h)$ in the two hierarchies. This point is explained in detail in [5].
It is tempting to offer a physical explanation of the identity of the of $\mathcal{Z}(h)$. In some way it must be connected with the scaling exponent of the probability distribution function of subset of velocity configurations with a given $h$. In other words, if the probability to find in a turbulent ensemble a solution $\left\{u_{n}\right\}$ of the unforced equations in the inviscid limit which is $P_{h}\left(\left\{u_{n}\right\}\right)$, then the probability to find a rescaled solution $\mathcal{R}(h, \rho)\left\{u_{n}\right\}$ is $\rho^{\mathcal{Z}(h)} P_{h}\left(\mathcal{R}(h, \rho)\left\{u_{n}\right\}\right)$. It should be stressed however that the issue of the rescaling properties of the ergodic measure of our model is far from being exhausted by this comment, and a lot of further research is needed to solidify and interpret it further. We leave this interesting issue for future research.

We reiterate that at this point $\rho$ and $h$ are free parameters, and $\mathcal{Z}(h)$ is an $n$-independent arbitrary function of $h$. We will see later that this freedom is restricted by the equations: although $\rho$ remains arbitrary, $h$ belongs to a natural interval, and the freedom of $\mathcal{Z}(h)$ is removed by a solvability condition for the hierarchy of equations.

As stressed before [2], the existence of the rescaling group $\mathcal{R}(h, \rho)$ for the linear set of equations suggests the existence of particular solutions that are characterized by $h$. In other words, we consider solutions denoted as $F_{n ; h}$ and $G_{m, n ; h}$ that solve equations on an $h$-slice, for 
example

$$
\begin{aligned}
& \frac{\partial}{\partial t_{1}} F_{2 ; h}\left(k_{n}, t_{1}, t_{2}\right)=\gamma_{a, n+1} F_{3 ; h}\left(k_{n+1} ; t_{2}, t_{1}, t_{1}\right) \\
& +\gamma_{b, n} F_{3 ; h}\left(k_{n} ; t_{1}, t_{2}, t_{1}\right)+\gamma_{c, n-1} F_{3 ; h}\left(k_{n-1} ; t_{1}, t_{1}, t_{2}\right),
\end{aligned}
$$

where the rescaling property of a particular solution $F_{n, h}$ is

$$
\mathcal{R}(h, \rho) F_{n, h}=\rho^{n h+\mathcal{Z}(h)} F_{n, h}\left(k_{m} ; t_{1}, \ldots, t_{n}\right) .
$$

The general solution $F_{n}$ of the hierarchical equations that we are interested in is naturally obtained as the weighted integral

$$
F_{n}\left(\left\{k_{m}\right\},\{t\}\right)=\int d \mu(h) F_{n, h}\left(\left\{k_{m}\right\},\{t\}\right),
$$

where $\left\{k_{m}\right\}=k_{m^{\prime}}, k_{m^{\prime \prime}}, \cdots$ and $\{t\}=t^{\prime}, t^{\prime \prime}, \cdots$ are the sets of $k$ - and $t$ - arguments of correlation functions $F_{n}$. The weight $d \mu(h) / d h$ may be, in principle, found from the boundary conditions, by matching the general solution in the inertial interval with non-universal part of $F_{n}$ in the energy containing interval.

The representation (44) follows rigorously from the rescaling symmetry of the problem and the linearity of the hierarchical equations. It offers a very useful connection to the multi-fractal model for anomalous scaling in turbulence that was introduced phenomenologically for simultaneous fully fused objects in [12]. In [5] it was realized that the multifractal model follows naturally from the equations of motion for the fully unfused multi-time correlation functions. Introduce the following dimensionless objects:

$$
\tilde{F}_{n, h}\left(\left\{\kappa_{m}\right\} ;\{\tau\}\right) \equiv \frac{1}{u_{0}^{n}}\left[\frac{\bar{k}_{m}}{k_{0}}\right]^{n h+\mathcal{Z}(h)} F_{n, h}\left(\left\{k_{m}\right\} ;\{t\}\right),
$$

where $\bar{k}_{m}$ is the geometric mean of the $\left\{k_{m}\right\}$, vectors involved in the correlation function: $k_{m_{1}}, k_{m_{2}}, \ldots$ Dimensionless $k$ - and $t$-arguments $\kappa$ and $\tau$ are defined as

$$
\kappa_{m_{j}} \equiv \frac{k_{m_{j}}}{\bar{k}}, \quad \tau_{j} \equiv \frac{t_{j}}{t\left(m_{j}, h\right)},
$$

where $t(m, h)$ is a characteristic time on the $m$-shell for the $h$-slice is

$$
t(m, h) \equiv \frac{1}{k_{0} u_{0}}\left(\frac{k_{m}}{k_{0}}\right)^{1-h} .
$$

Finally the integral (44) is written as

$$
F_{n}(\{k\},\{t\})=u_{0}^{n} \int d \mu(h)\left[\frac{k_{0}}{\bar{k}_{m}}\right]^{n h+\mathcal{Z}(h)} \tilde{F}_{n, h}(\{\kappa\},\{\tau\}) .
$$

It is easy to recognize that this formula is a natural (multi-time, multi-point) generalization of the multifractal representation of structure functions if $\mathcal{Z}(h)=$ $3-D(h)$ where $D(h)$ is the fractal dimension of the set of points that support an Hölder exponent $h$ [4]. The function $\mathcal{Z}(h)$ is related to the scaling exponents of the structure functions $\zeta_{n}$ via the saddle point requirement

$$
\zeta_{n}=\min _{h}[n h+\mathcal{Z}(h)] \text {. }
$$

This identification stems from the fact that the integral in (48) is computed in the limit $\left(k_{0} / \bar{k}_{m}\right) \rightarrow \infty$ via the steepest decent method. Neglecting logarithmic corrections one find that $F_{n} \propto\left(k_{0} / \bar{k}_{m}\right)^{\zeta_{n}}$.

\section{Z-COVARIANT CONTROLLED CLOSURE}

In [5] we introduced a closure scheme that exploits the rescaling symmetry of the hierarchy of equation on $h$ slices. This closure turns out to be somewhat different from traditional closures. Since the first attempts in the 40 's it has been customary to close the set of equations in the most natural way, that is by expressing high order correlation functions in terms of lower order ones. Generally such procedures do not preserve the fundamental rescaling symmetry (38). For example, if one tries to estimate $F_{4}$ as $F_{2}^{2}$, it would require $\mathcal{Z}(h)=0$. We propose that attempts to close the hierarchy without respecting the rescaling symmetry (with $\mathcal{Z}(h) \neq 0$ ) necessarily lead to fundamental mistakes of this type, ruining the possibility of finding anomalous exponents.

The minimal requirements for an acceptable closure should be that realizability conditions are guaranteed: we cannot have negative PDFs and negative energy spectra. In addition, we want to preserve the Galilean and rescaling symmetries of the original equations. We therefore propose a closure scheme in which both these requirements are fulfilled. Realizability is guaranteed because in our model we have small parameters, i.e. $1 / N$ and $\epsilon$ that are used to control the closure, making the neglected terms vanishingly small compared with those retained in the limit $1 / N \rightarrow 0$ and $\epsilon \rightarrow 0$. The second requirement is met by expressing higher order correlation (fourth order quantities in the lowest order closure) in term of lower order ones in such a way that the rescaling symmetry (41) in an $h$-slice is preserved. In particular all our closure steps are $\mathcal{Z}(h)$ covariant, in the sense that all the terms in the resulting equations have the same $\mathcal{Z}(h)$ dependence. As a consequence the equations are neutral to power counting, and $\mathcal{Z}(h)$ is computed from coefficients rather than powers. In fact $\mathcal{Z}(h)$ is found from solvability conditions as is demonstrated below.

The procedure developed in the rest of this paper is a closure at the level of $F_{4 ; h}$. We are going to represent $F_{4 ; h}$ in terms of the lower order quantities, the two point quantities $F_{2 ; h}$ and $G_{1,1 ; h}$, and the three point quantities 
$F_{3 ; h}, G_{1,2 ; h}$ and $G_{2,1 ; h}$. There is a part of $F_{4 ; h}$ that cannot be represented in terms of these lower order quantities, but we will show that this part is of lower order in $\epsilon$, in fact of $O\left(\epsilon^{6}\right)$ whereas the retained terms are of $O\left(\epsilon^{4}\right)$. In order to show this explicitly we will have to consider the closure at the level of $F_{5 ; h}$, representing $F_{5 ; h}$ in terms of all the lower order 2-point, 3-point and 4-point objects.

In representing higher order quantities in terms of lower order ones there are many different combinations of low order quantities which appear in any given higher order one. In fact all the topologically possible combinations are allowed. To facilitate the analysis it is natural to represent these by diagrams. In making three point objects from two point ones, there appear three different ways of combining the two point objects. These are represented by "vertices" which are denoted below as $A_{h}, B_{h}$ and $C_{h}$. In perturbation theories such vertices are commonly represented in terms of infinite series. In our approach (as in a fully resumed diagrammatic theory) these vertices are solved for exactly in terms of the three-point objects on an $h$-slice. It turns out, once the theory is written down, that these vertices appear always in the same combinations with the 2-point Green's functions $G_{1,1 ; h}$ which never appears alone. We thus work in terms of three newly defined objects that we call "triplices". The $A$-triplex is the vertex $A_{h}$ linked to $G_{1,1 ; h}$, the $B$-triplex is the vertex $B_{h}$ linked to two $G_{1,1 ; h}$ 's, and the $C$-triplex is the vertex $C_{h}$ linked to three $G_{1,1 ; h}$ 's. We reiterate that at no point do we need to sum infinite series.

At the end of our procedure we will pose equations of motion for the $F_{2 ; h}$ and the three triplices, which are closed upon themselves and are valid to $O\left(\epsilon^{4}\right)$. A simplified version which includes only one $A$-triplex is shown in Fig. 5 . This simplified set of equations is analyzed in the sequel, and we show that as a function of $\epsilon$ it predicts a transition from normal scaling to anomalous scaling. The reader who is not fascinated by the technical details can skip Sect. VI, and observe the appearance of solvability conditions in Sect. VIII

\section{TECHNICAL DETAILS OF DERIVING THE CLOSURE EQUATIONS}

\section{A. Definition of the triplex objects $T_{A ; h}, T_{B ; h}, T_{C ; h}$}

As a preparatory step for the closure procedure we introduce three types of triplices: A-triplex $T_{A ; h}$, B-triplex $T_{B ; h}$ and C-triplex $T_{C ; h}$. Triplices are functions of three $k$ - and three $t$ arguments, say $k, k^{\prime}, k^{\prime \prime}$ and $t, t^{\prime}, t^{\prime \prime}$. As any three point objects of the Sabra-shell model they differ from zero only for three consecutive shell numbers, say $m-1, m$ and $m+1$. The C-triplex is invariant under all permutations of points, therefore it is enough to

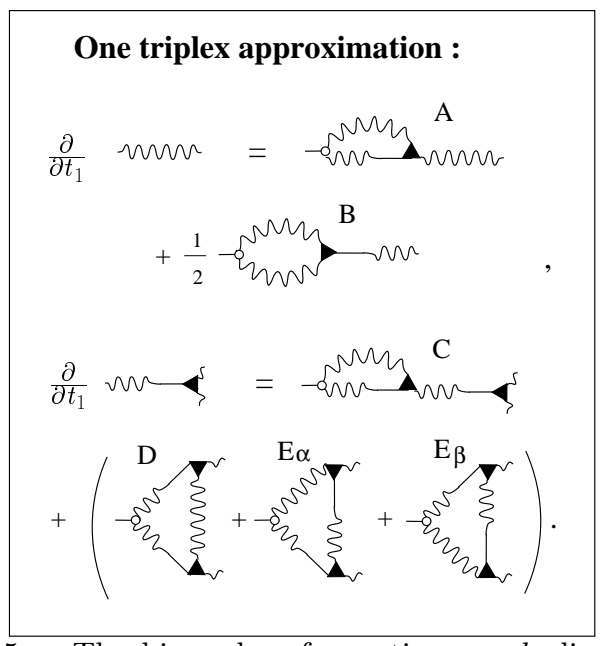

FIG. 5. The hierarchy of equations on $h$-slice after the level-4 closure. For the beginning we did not consider here contributions of $B$ - and $C$ - triplices. This allows to reduce a system of four equations to the system of just two equations for $F_{2 ; h}$ (two top lines) and for $A$-triplex (two bottom lines). Equation for $A$-triplex follows for equation in Fig. 8 after disregarding the empty square term and multiplying the result on the right by two inverse Green's' functions. f:closed1

mention only one of the $k$-arguments, corresponding, for example to the middle shell $k_{m}$. So, we have $T_{C ; h}=$ $T_{C ; h}\left(k_{m} ; t, t^{\prime}, t^{\prime \prime}\right)$. The convention here is that the first time argument $t$ corresponds to the smallest shell $m-1$, the second $t^{\prime}$ to the middle shell $m$ and the last $t^{\prime \prime}$ to the $m+1$-shell. The same convention is chosen for the $A$ - and $B$-triplices, the only difference is that these two are invariant only under permutation of two points, the third one is special. Therefore we have three different $A$ and $B$-triplices (and one $C$-triplex) denoted as:

$$
T_{A ; h}^{(+1)}, T_{A ; h}^{(-1)}, T_{A ; h}^{(0)} ; \quad T_{B ; h}^{(+1)}, T_{B ; h}^{(-1)}, T_{B ; h}^{(0)} ; \quad T_{C ; h} .
$$

The superscript $(+1)$ means here that the $k$-argument of the special shell is $k_{m+1},(-1)$ corresponds to $k_{m-1}$ and (0) - to $k_{m}$. These objects are defined in terms of the following seven three point functions (with the same convention concerning the arguments, see their definitions (A4)- A7):

$$
G_{1,2 ; h}^{(+1)}, G_{1,2 ; h}^{(-1)}, G_{1,2 ; h}^{(0)} ; \quad G_{2,1 ; h}^{(+1)}, G_{2,1 ; h}^{(-1)}, G_{2,1 ; h}^{(0)} ; \quad F_{3 ; h} .
$$

Three A-triplices $T_{A ; h}$ are defined in terms of the three corresponding (with the same superscript) Green's functions $G_{1,2 ; h}$, which describe the response of the velocity $v_{m_{1}}$ to two forcing factors on the shells $m_{2}$ and $m_{3}$ defined by Eq. A7). In terms of $G_{1,2 ; h}$ we have: 


$$
G_{1,2 ; h}^{(0)}\left(k_{m}, t_{1}, t_{2}, t_{3}\right)=\int_{t_{1}}^{\infty} d t_{1}^{\prime} \int_{t_{3}}^{\infty} d t_{3}^{\prime} T_{A ; h}^{(0)}\left(k_{m} ; t_{1}^{\prime}, t_{2}, t_{3}^{\prime}\right) G_{1,1 ; h}\left(k_{m-1} ; t_{1}^{\prime}-t_{1}\right) G_{1,1 ; h}\left(k_{m+1} ; t_{3}^{\prime}-t_{3}\right)
$$

Similar equations serve to define the triplices $T_{A ; h}^{+1}$ and $T_{A ; h}^{(-1)}$ simply by replacing on the LHS the appropriate response functions and by the required changes of the time integration on the RHS:

$$
\begin{aligned}
& G_{1,2 ; h}^{(+1)}\left(k_{m}, t_{1}, t_{2}, t_{3}\right)=\int_{t_{1}}^{\infty} d t_{1}^{\prime} \int_{t_{2}}^{\infty} d t_{2}^{\prime} T_{A ; h}^{(+1)}\left(k_{m} ; t_{1}^{\prime}, t_{2}^{\prime}, t_{3}\right) G_{1,1 ; h}\left(k_{m-1} ; t_{1}^{\prime}-t_{1}\right) G_{1,1 ; h}\left(k_{m} ; t_{2}^{\prime}-t_{2}\right) \\
& G_{1,2 ; h}^{(-1)}\left(k_{m}, t_{1}, t_{2}, t_{3}\right)=\int_{t_{2}}^{\infty} d t_{2}^{\prime} \int_{t_{3}}^{\infty} d t_{3}^{\prime} T_{A ; h}^{(-1)}\left(k_{m} ; t_{1}, t_{2}^{\prime}, t_{3}^{\prime}\right) G_{1,1 ; h}\left(k_{m} ; t_{2}^{\prime}-t_{2}\right) G_{1,1 ; h}\left(k_{m+1} ; t_{3}^{\prime}-t_{3}\right) .
\end{aligned}
$$

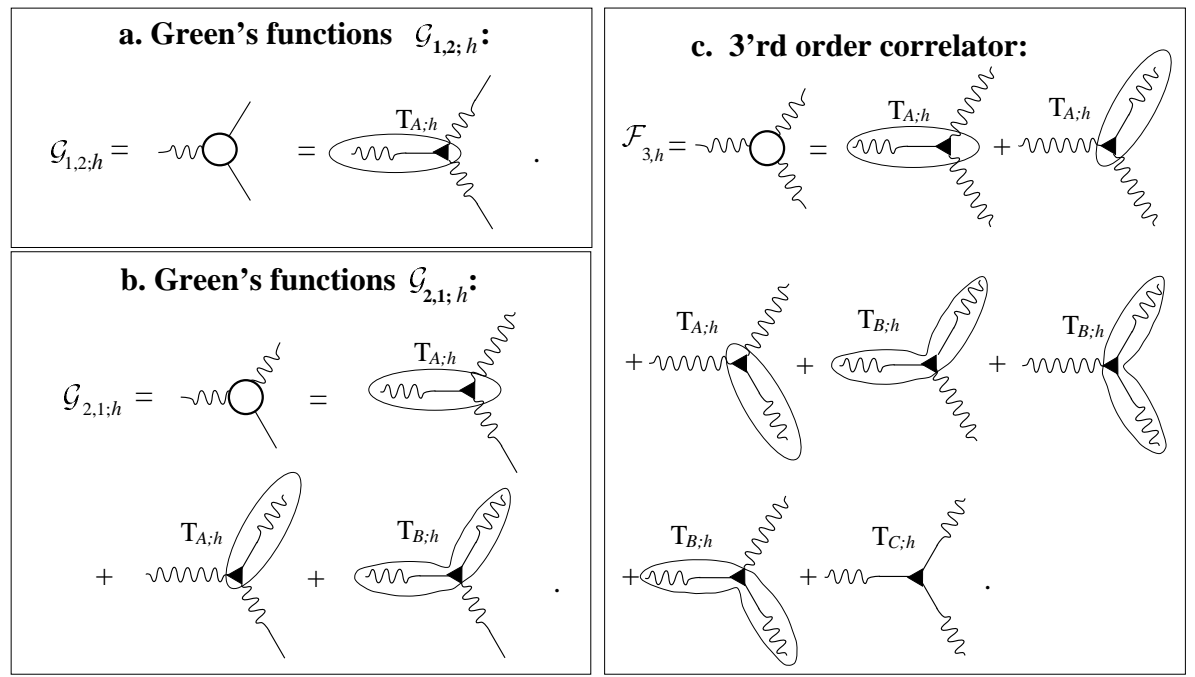

FIG. 6. Exact representation of the Green's functions $G_{2,1 ; h}$ and $G_{1,2 ; h}$ (Panels a and b) and correlation function $F_{3 ; h}$ in terms of the vertex $A$ and the Green's' functions $G_{1,1}$. In our approach these serve as the definitions of triplices $T_{A ; h}, T_{B ; h}, T_{C ; h}$, on an $h$ slice via $F_{2 ; h}$ and $G_{2,1 ; h}, G_{1,2 ; h}, F_{3 ; h}$.

Now let us have a look in Fig. $6 \mathrm{~b}$ at the representation of the Green's functions $G_{2,1}$ in terms of the two triplices $A, B$, the correlation function $F_{2}$ and the Green's' functions $G_{1,1}$. This serves to define the $B$-triplex. The explicit analytic definition of $T_{B ; h}^{(0)}$ triplex is:

$$
\begin{gathered}
G_{2,1 ; h}^{(0)}\left(k_{m}, t_{1}, t_{2}, t_{3}\right)=\int_{t_{2}}^{\infty} d t_{2}^{\prime}\left\{\int_{0}^{\infty} d t_{3}^{\prime} T_{A ; h}^{(0)}\left(k_{m} ; t_{1}, t_{2}^{\prime}, t_{3}^{\prime}\right) F_{2 ; h}\left(k_{m+1} ; t_{3}^{\prime}-t_{3}\right) G_{1,1 ; h}\left(k_{m} ; t_{2}^{\prime}-t_{2}\right)+\int_{0}^{\infty} d t_{1}^{\prime}\right. \\
\left.\times T_{A ; h}^{(+1)}\left(k_{n} ; t_{1}^{\prime}, t_{2}^{\prime}, t_{3}\right) G_{1,1, i h}\left(k_{m} ; t_{2}^{\prime}-t_{2}\right) F_{2 ; h}\left(k_{m-1} ; t_{1}^{\prime}-t_{1}\right)\right\}+\int_{0}^{\infty} d t_{2}^{\prime} T_{B ; h}^{(0)}\left(k_{n} ; t_{1}, t_{2}^{\prime}, t_{3}\right) G_{1,1 ; h}\left(k_{m-1} ; t_{2}^{\prime}-t_{2}\right)
\end{gathered}
$$

Similar equations for $T_{B ; h}^{(-1)}$ and $T_{B ; h}^{+1}$ can be derived by using $G_{2,1 ; h}^{(-1)}$ and $G_{2,1 ; h}^{(+1)}$. Analogously, Fig. $6 c$ allows one to define $C$ triples:

$$
\begin{aligned}
& F_{3 ; h}\left(k_{m}, t_{1}, t_{2}, t_{3}\right)=\int_{0}^{\infty} d t_{2}^{\prime} \int_{0}^{\infty} d t_{3}^{\prime} T_{A ; h}^{(-1)}\left(k_{m} ; t_{1}, t_{2}^{\prime}, t_{3}^{\prime}\right) F_{2 ; h}\left(k_{m} ; t_{2}^{\prime}-t_{2}\right) F_{2 ; h}\left(k_{m+1}, t_{3}^{\prime}-t_{3}\right) \\
& +\int_{0}^{\infty} d t_{1}^{\prime} \int_{0}^{\infty} d t_{3}^{\prime} T_{A ; h}^{(0)}\left(k_{m} ; t_{1}^{\prime}, t_{2}, t_{3}^{\prime}\right) F_{2 ; h}\left(k_{m-1}, t_{1}^{\prime}-t_{1}\right) F_{2 ; h}\left(k_{m+1}, t_{3}^{\prime}-t_{3}\right) \\
& +\int_{0}^{\infty} d t_{1}^{\prime} \int_{0}^{\infty} d t_{2}^{\prime} T_{A ; h}^{(+1)}\left(k_{m} ; t_{1}^{\prime}, t_{2}^{\prime}, t_{3}\right) F_{2 ; h}\left(k_{m-1}, t_{1}^{\prime}-t_{1}\right) F_{2 ; h}\left(k_{m}, t_{2}^{\prime}-t_{2}\right) \\
& +\int_{0}^{\infty} d t_{1}^{\prime} T_{B ; h}^{(-1)}\left(k_{m}, t_{1}^{\prime}, t_{2}, t_{3}\right) F_{2 ; h}\left(k_{m-1}, t_{1}^{\prime}-t_{1}\right)+\int_{0}^{\infty} d t_{2}^{\prime} T_{B ; h}^{(0)}\left(k_{m}, t_{1}, t_{2}^{\prime}, t_{3}\right) F_{2 ; h}\left(k_{m}, t_{2}^{\prime}-t_{2}\right) \\
& +\int_{0}^{\infty} d t_{3}^{\prime} T_{B+1, h}^{(0)}\left(k_{m}, t_{1}, t_{2}, t_{3}^{\prime}\right) F_{2 ; h}\left(k_{m+1}, t_{3}^{\prime}-t_{3}\right)+T_{C ; h}\left(k_{m} ; t_{1}, t_{2}, t_{3}\right) .
\end{aligned}
$$




\section{B. Representation of the 4-point functions}

This theory has four different fundamental 4-point statistical objects: the correlation function $F_{4 ; h}$ and three non-linear Green's' functions: $G_{3,1 ; h}(\mathrm{~A} 9), G_{2,2 ; h}$ (A10), and $G_{1,3 ; h}$. These objects are shown in Fig. a as circles with four tails: four wavy tails for $F_{4 ; h}$, three wavy and one straight tail for $G_{3,1 ; h}$, etc. As the 3-point objects, also the 4-point objects can be represented as combination of lower order 2 and 3-point objects, and in addition they have a piece that cannot be so represented. Consider Fig.7. For every 4-point statistical object we show the part obtained from 2-point objects, which is the Gaussian decomposition, a part that is given in terms of 2-point objects and 3-point vertices, and finally the part that calls for the introduction of 4-point vertices which are represented by empty squares and denoted by $D$. There are four different $D$-vertices, depending on the nature of the tails connecting them. As in the case of the 3-point vertices, the 4-point vertices that can be solved for in terms of the 4-point statistical objects. The figures offer a strong motivation for the graphic representation. Analytically the equation for $F_{4 ; h}$ consist of 63 terms, Eqs. for $G_{3,1 ; h}$ has 31 terms, etc. The graphic notation is sufficient for all our future considerations.

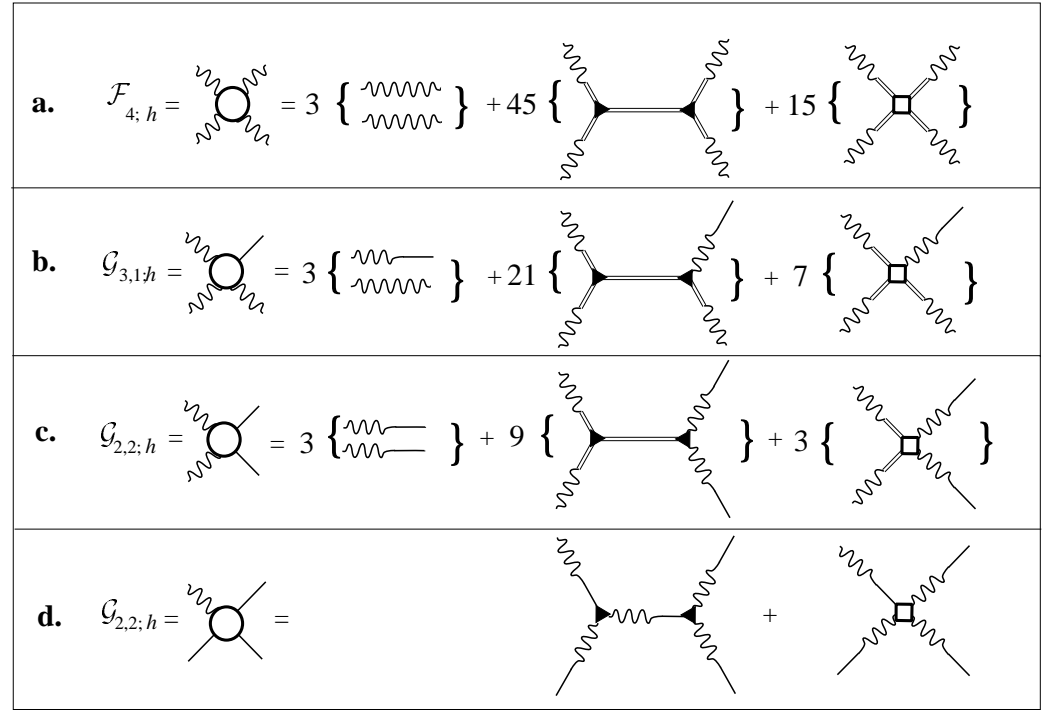

FIG. 7. Compact representation of the 4th-order statistical objects in terms of triplices and irreducible 4 th order vertices (empty squares). Double lines denote either wavy or straight lines. Thus a short wavy and short double line denote either pair correlator (long wavy line, when the short double line denotes a wavy one) or the Green's' function (short wavy and short straight line, when the double line denotes a straight one). One understands a long double line as consisting of two short double line. Therefore a long double line may denote 3 versions of 2-point objects: the 2-point correlation function and 2 orientations of the Green's functions. The long double line is not allowed to be made of two short straight lines. The different topologies are counted in these figures, for example, Panel a one has 3 different Gaussian decompositions of $F_{4 ; h}$, 45 different contributions in terms of triplices and 15 contributions in terms of 4-point vertices.
In particular, by substituting these representations on the RHS of the hierarchy of equation for the 3-point objects (Panel $\mathrm{c}$ and bottom lines of the Panels a and $\mathrm{b}$ of Fig. (1) we get Equations for the 3-point objects in terms of the 2,3-point objects and 4-point vertices. An example of such an equation is given in Fig. 8.

\section{Closure on the level of 4 th order objects}

The simplest closure which may already predict anomalous scaling is very simple, and is obtained by neglecting the empty squares in all the equations of motion for 2- and 3-point objects. Below we show that in this approximation we account exactly for all terms up to $O\left(\epsilon^{4}\right)$ and neglect terms of $O\left(\epsilon^{6}\right)$ and smaller. The resulting system of equations can be exactly reduced to a system of four evolution equations for $F_{2 ; h}$ and the three triplices. A simplified version of these equations involving the A-triplex only is shown in Fig. 5 .

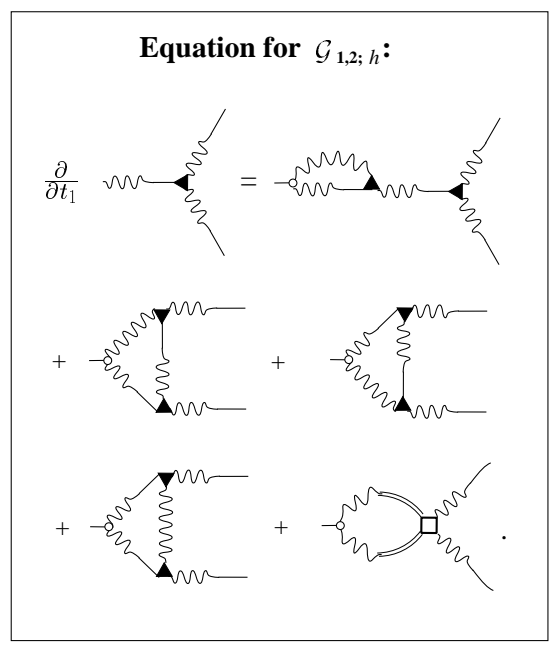

FIG. 8. Equation for $G_{1,2 ; h}$. For simplicity we did not display here the terms with $B$ - and $C$-triplices. Topologically they look like the exhibited terms with the $A$-triplex.

\section{Closure on the level of 5th order objects}

In order to formulate this closure consider four sets of hierarchical equations:

- The first set consists of 3 evolution equations for the correlation functions $F_{2 ; h}, F_{3 ; h}$ and $F_{4 ; h}$. These equations contain on their RHS $F_{3 ; h}, F_{4 ; h}$ and $F_{5 ; h}$ respec- 
tively.

- The second set consists of 3 equations for the Green's' functions $G_{1,1 ; h}, G_{2,1 ; h}$, and $G_{3,1 ; h}$, having on their RHS the $G_{2,1 ; h}, G_{3,1 ; h}$, and $G_{4,1 ; h}$.

- The third set consists of 2 equations for the Green's' functions $G_{1,2 ; h}$ and $G_{2,2 ; h}$ in terms of $G_{2,2 ; h}$ and $G_{3,2 ; h}$.

- The fourth set has just one equation for the Green's function $G_{1,3 ; h}$ in terms of $G_{2,4 ; h}$.

The new objects that appear at this level are the four 5th order functions $F_{5 ; h}, G_{2,3 ; h}, G_{3,2 ; h}$ and $G_{4,1 ; h}$ which appear in the RHS of the last equation in each of the four sets. Like the 4 th-order objects they may be presented as a sum of reducible and irreducible parts, the latter being related to a new set of 5 th order vertices that can be represented graphically as empty pentagons. The reducible part is a sum of all the topologically allowed contributions made from lower order objects: $F_{2 ; h}, G_{1,1 ; h}$, three triplices and four 4 th order vertices $D_{4-n, n ; h}$. The irreducible part of the statistical objects is, by definition, what is not reducible. The Closure on level of 5th-order objects is obtained by disregarding all the irreducible contributions in 5th-order objects.

We are not going to study explicitly the equations obtained from the closure at the level of the 5th order objects. We use this level to justify the relative smallness of what was neglected in the closure on the level of the 4th order objects. To this aim we derive now an exact equation for the empty squares that does not contain any time derivative. This is done as follows: Compute $\partial F_{4 ; h} / \partial t_{1}$ using the representation of $F_{4 ; h}$ via $D_{4-n, n ; h}$ and 2 nd order objects, shown in Fig. 7. For the time derivatives of the 2 nd order objects use the corresponding evolution equations involving $F_{3 ; h}$ and the third order Green's functions. The latter in their turn may be expressed exactly via 3rd order vertices (black triangles). On the other hand we have the evolution equation for $\partial F_{4 ; h} / \partial t_{1}$ which relates it to $F_{5 ; h}$. Now $F_{5 ; h}$ can be presented as a sum of reducible contributions (made from lower order objects) and an irreducible part, which is presented via empty pentagons. Equating the formulae for $\partial F_{4 ; h} / \partial t_{1}$ one gets after some tedious algebra an exact linear equation for the 4 th order vertices in terms of lower order objects and the 5 th order empty pentagons. This equation is displayed in Fig. 9. The closure on the 5th level amounts to discarding the empty pentagons, leading to a linear inhomogeneous equation for the empty squares. This equation serves for the order of magnitude estimates presented in the next section.

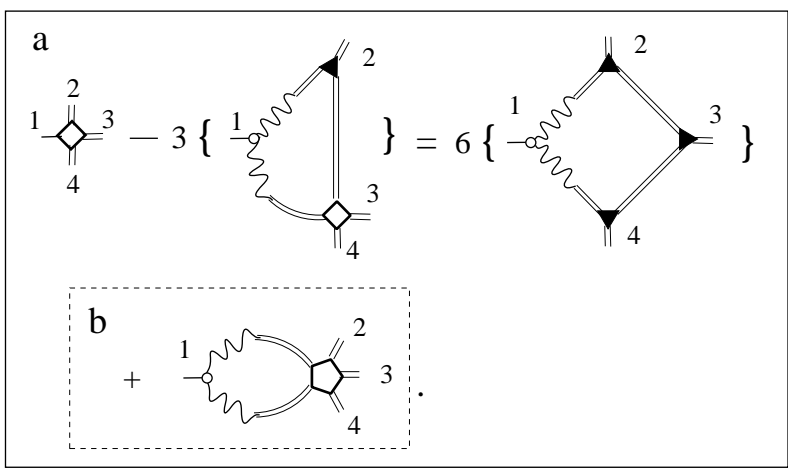

FIG. 9. Exact equation for $D_{n, 4-n}^{\mathrm{ir}}$ (empty square).

\section{ORDERS OF MAGNITUDE}

In this Section we explain how the small parameter $\epsilon$ controls the sequential steps in the closure procedure in the $(N, \epsilon)$-model. In particular, we address the empty square in Fig. 8 that is being discarded in the closure on the level of 4 th order objects, showing that it is of lower order in $\epsilon$ compared to the terms that are retained in the $\operatorname{limit} N \rightarrow \infty$.

First, recall that initially all our objects had "Fourier space" indices $\alpha, \beta, \gamma, \ldots$ and spinor indices $\sigma, \sigma^{\prime}, \sigma^{\prime \prime} \ldots$ The tensorial correlation functions $F_{2 ; \sigma, \sigma^{\prime}}^{\alpha}, F_{3, \sigma, \sigma^{\prime}, \sigma^{\prime \prime}}^{\alpha, \alpha^{\prime}, \alpha^{\prime \prime}}$ and $F_{4, \sigma, \sigma^{\prime} \ldots}^{\alpha, \alpha^{\prime} \ldots}$ are defined by Eqs. (23) - (25) and the tensorial Green's functions of the 2nd, 3rd and 4th order are defined by Eqs. (A2)-(A5). Equations (33)- (35) introduce the scalar objects $F_{2}, F_{3}$ and $F_{4}$ as sums over tensor indices. For these objects we derived scalar equations and that were represented diagrammatically. Of course, all the diagrammatic equations (including equations on an $h$-slice) discussed so far may be understood also as equations for the tensor objects with explicit $\alpha, \beta, \gamma, \ldots$ and $\sigma, \sigma^{\prime}, \sigma^{\prime \prime} \ldots$ indices. In this case each equation (or diagram) for the tensor objects includes summations over dummy Fourier and spinor indices for all the intervening objects, (vertices, triplices, etc.), in the body of the diagrams. This calls for definitions of tensorial triplices and fourth and fifth order vertices. In particular, each triplex is understood as a sum similar to (34) of objects depending on $\alpha, \beta$ 's. For example:

$$
\begin{gathered}
T_{A ; h}^{(0)}\left(k_{n} ; t_{1}, t_{2}, t_{3}\right)=\frac{1}{N} \sum_{a, \beta, \gamma}\left[\Delta_{\alpha, \beta+\gamma}+\Delta_{\alpha+N, \beta+\gamma}\right. \\
\left.+\Delta_{\alpha, \beta+\gamma+N}\right] T_{A ; h}^{(0) \alpha, \beta, \gamma}\left(k_{n} ; t_{1}, t_{2}, t_{3}\right),
\end{gathered}
$$

where the sums on $\sigma$ 's have already been performed. The objects $T_{A}^{\alpha, \beta, \gamma}$ are obtained from tensorial equations identical to 52 56), but with the 2 nd and 3rd order objects having the appropriate Greek indices. Having done so it follows that $T_{A ; h}^{\alpha, \beta, \gamma}$ is of order $O(1 / \sqrt{N})$ : this stems from the fact that $F_{3 ; h}^{\alpha, \beta, \gamma}$ is of order $O(1 / \sqrt{N})$ and $F_{2 ; h}^{\alpha}$ 
and $G_{1,1 ; h}^{\alpha \alpha}$ are $O(1)$. In other words, $T_{A ; h}^{\alpha, \beta, \gamma}$ has the same order magnitude and the same tensorial structure as the 3 -point correlation function $F_{3 ; h}^{\alpha, \beta, \gamma}$. Again, Eq. (57) can be inverted to give:

$$
T_{A ; h}^{(0) \alpha, \beta, \gamma}=\Phi^{-\alpha,-\beta,-\gamma} T_{A ; h}^{(0)},
$$

where $T_{A ; h}^{(0)}$ is a function $O(1)$. Similar relationships may be written for the other triplices.

Using this information we can discuss the order of magnitude of the terms in the equation for $G_{1,2 ; h}$ exhibited in Fig. 8. All the following considerations apply similarly to the equations for $F_{3 ; h}$ and $G_{2,1 ; h}$. In fact the order of magnitude of the different terms in the equations does depend only on the topology of the graphs and not on the kind of 2-point functions.

The equation in Fig. 8 has three types of contributions. The first one on the RHS has a "simple" loop (made of 2 lines representing 2 nd order objects). The next three terms have a loop made from 3 lines ("triangular" loop) and the last term has a 4th order vertex, an empty square.

The term with the simple loop has two sums over Greek indices, each having a product of two complex conjugate $\Phi$ 's (three factors $\Phi$ 's coming from the three vertices and one coming from the definition of $\left.G_{1,2 ; h}\right)$. Accordingly this term is of the order of unity, as the LHS. In the triangular loops we also have two sums on Greek indices of four factors of $\Phi$. In this case the term will be multiplied by the following factor

$$
\begin{aligned}
& \frac{1}{N} \sum_{\alpha \beta \delta} \Phi^{\alpha+\beta, \alpha, \beta} \Phi^{\alpha, \delta, \alpha-\delta} \Phi^{\beta,-\delta, \beta+\delta} \Phi^{\alpha-\delta, \alpha+\beta,-\beta-\delta} \\
= & \frac{1}{N^{3}} \sum_{\alpha \beta \delta}\left(\epsilon+\sqrt{1-\epsilon^{2}} \Psi^{\alpha+\beta, \alpha, \beta}\right)\left(\epsilon+\sqrt{1-\epsilon^{2}} \Psi^{\alpha, \delta, \alpha-\delta}\right) \\
\times & \left(\epsilon+\sqrt{1-\epsilon^{2}} \Psi^{\beta,-\delta, \beta+\delta}\right)\left(\epsilon+\sqrt{1-\epsilon^{2}} \Psi^{\alpha-\delta, \alpha+\beta,-\beta-\delta}\right) .
\end{aligned}
$$

The four factors $\Psi$ do not appear as couples of complex conjugates. As a result, after averaging over the randomness and sending $N \rightarrow \infty$ all the terms that present a random phase in (59) will go to zero and only the factor $\epsilon^{4}$ will survive. The conclusion, which is of fundamental importance for the solution of our model, is that terms with triangular loops are smaller than terms with a simple loop by a factor of $O\left(\epsilon^{4}\right)$. This is the first place that $\epsilon$ appears as an important parameter that distinguishes the order of magnitude of competing terms. We reiterate that usually, without the introduction of $\epsilon$, all the diagrams that one obtains in the context of turbulent statistics are of the same order.

The second important role of the small parameter $\epsilon$ is to make the last term with an empty square even smaller than the terms just analyzed. We show that it is of the order of $\epsilon^{6}$. In order to see this, consider the equation for the empty squares exhibited in Fig. 9. The irreducible part of $F_{5 ; h}$ is related here to the empty pentagon. At this point we discard the empty pentagon without much ado, and find the order of magnitude of the empty square. Of course, this step should be justified by estimating next the order of the empty pentagon, which can be done by going to the closure on the level of 6 th order objects [with the result that the term with the empty pentagon is smaller than the other terms in this equations by a factor of $O\left(\epsilon^{2}\right)$. This term is of $\left.O\left(\epsilon^{8}\right)\right]$.

After discarding the empty pentagon we have an equation for the empty square in terms of itself and of lower order objects. This equation corresponds to the top line in Fig. 9. On the RHS we have objects with four vertices in one loop. Accordingly they are of $O\left(\epsilon^{4}\right)$. The LHS has an operator that acts on our empty square. The operator contains unity, and is therefore estimated to be of the $O(1)$. This is in fact a dangerous point. We are not guaranteed a priori that the empty square is not a zero mode of this operator. We are going to make an assumption that this is not the case. This assumption cannot be justified before the end of the calculation. In principle, one needs to proceed under this assumption, compute explicitly the 2nd and 3rd order objects, and justify the assumption a posteriori by a direct substitution and calculation.

Making this assumption we conclude that the term including the empty square in Fig. 8 is of $O\left(\epsilon^{6}\right)$ and it can be neglected with impunity. This leaves us with the equation represented in Fig. 5. Previously we were satisfied with this graphic representation but now we need to discuss its analytic form. In Appendix B we display the algebraic form in the $\omega$-representation in full. For the scale invariant functions (B18) these equations may be written as follows:

$$
\begin{aligned}
-\left[i \Omega+\tilde{\sigma}_{h}(\Omega)\right] & \tilde{\mathcal{F}}_{2 ; h}(\Omega)=\tilde{\mathcal{B}}_{h}(\Omega), \\
\mu\left[i \Omega_{+}+\tilde{\sigma}^{*}\left(\Omega_{+}\right)\right] & \tilde{\mathcal{T}}_{A ; h}^{(+1)}\left(\Omega_{-}, \Omega_{0}\right) \\
& =\epsilon^{4}\left[\tilde{\mathcal{D}}_{h}^{(+1)}\left(\Omega_{-}, \Omega_{0}\right)+\tilde{\mathcal{E}}_{h}^{(+1)}\left(\Omega_{-}, \Omega_{0}\right)\right], \\
-\left[i \Omega_{0}+\tilde{\sigma}_{h}\left(\Omega_{0}\right)\right] & \tilde{\mathcal{T}}_{A ; h}^{(0)}\left(\Omega_{-}, \Omega_{+}\right) \\
& =\epsilon^{4}\left[\tilde{\mathcal{D}}_{h}^{(0)}\left(\Omega_{-}, \Omega_{+}\right)+\tilde{\mathcal{E}}_{h}^{(0)}\left(\Omega_{-}, \Omega_{+}\right)\right], \\
-\left[i \Omega_{-}+\tilde{\sigma}\left(\Omega_{-}\right)\right] & \tilde{\mathcal{T}}_{A ; h}^{(-1)}\left(\Omega_{0}, \Omega_{+}\right) \\
& =\epsilon^{4}\left[\tilde{\mathcal{D}}_{h}^{(-1)}\left(\Omega_{0}, \Omega_{+}\right)+\tilde{\mathcal{E}}_{h}^{(-1)}\left(\Omega_{0}, \Omega_{+}\right)\right] .
\end{aligned}
$$

Here $\tilde{\mathcal{F}}_{2 ; h}$ is a dimensionless 2 nd order correlation functions, $\tilde{\mathcal{T}}_{A ; h}^{(\ell)}$ are dimensionless triplices. The term with the mass operator $\tilde{\sigma}$ originates from the diagram $\mathrm{A}$ in the equation for $\tilde{\mathcal{F}}_{2 ; h}$ and from diagram $\mathrm{C}$ in the Eqs. for $\tilde{\mathcal{T}}_{A ; h}^{(\ell)}$. The terms $\tilde{\mathcal{E}}_{h}^{(\ell)}$ and $\tilde{\mathcal{D}}_{h}^{(\ell)}$, with a factor $\epsilon^{4}$ in front, originate from the triangle diagrams $\mathrm{D}$ and $\mathrm{E}$ in Fig. 8. Analytical forms of these functions are given in Appendix B. 


\section{PRELIMINARY ANALYSIS OF THE SOLVABILITY CONDITIONS}

Equations (60)- 63), which are exact in the limit $\epsilon \rightarrow$ 0 , are nonlinear integral equations for one function of one frequency $\tilde{\mathcal{F}}_{2 ; h}(\Omega)$ and three functions $\tilde{\mathcal{T}}_{A ; h}^{(\ell)}$ (for $\ell=0$ and $\ell= \pm 1$ ) of two frequencies. A complete analysis of these equations is very difficult and beyond of the scope of this paper. In this section we will only demonstrate how the solvability conditions of these equations may determine the function $\mathcal{Z}(h)$. For the purpose of this demonstration it is sufficient to restrict ourselves to the "one-triplex reduction" of Eqs. (60)- (63). Considering only the two equations for $\tilde{\mathcal{F}}_{2 ; h}$ and $\tilde{\mathcal{T}}_{A ; h}^{(0)}$, we substitute on the RHS of these equations $\tilde{\mathcal{T}}_{A ; h}^{(0)}$ instead of $\tilde{\mathcal{T}}_{A ; h}^{( \pm 1)}$ :

$$
\begin{aligned}
&-\left[i \Omega+\tilde{\sigma}_{h}(\Omega)\right] \tilde{\mathcal{F}}_{2 ; h}(\Omega)=\tilde{\mathcal{B}}_{h}(\Omega), \\
&-\left[i \Omega_{0}+\tilde{\sigma}_{h}\left(\Omega_{0}\right)\right] \tilde{\mathcal{T}}_{A ; h}^{(0)}\left(\Omega_{-}, \Omega_{+}\right) \\
&=\epsilon^{4}\left[\tilde{\mathcal{E}}_{h}^{(0)}\left(\Omega_{-}, \Omega_{+}\right)+\tilde{\mathcal{D}}_{h}^{(0)}\left(\Omega_{-}, \Omega_{+}\right)\right] .
\end{aligned}
$$

Clearly, neglecting the difference between triplices with different $\ell$ is an uncontrolled step which can (and will) be improved in future work.

Given a set of integral equations (64), (65) it is customary to expand the unknown functions in an appropriate complete set of functions, and to project the resulting expanded form on each function in the set separately. In this way one generates a countable set of algebraic equations. The least automatic step in this procedure is the choice of the complete set of functions. By choosing the low order functions in the basis set to represent in some sense the properties of the expected solutions, one can hope that a truncated set may serve as well. It is of course also very helpful if the used set of functions is simple enough to allow as much analytic integration as possible. To find a good compromise between these requirements we suggest to use a set of functions:

$$
f_{0}(\Omega)=\frac{1}{\Omega+i \gamma}, \ldots \quad f_{n}(\Omega)=\frac{d^{n}}{d \gamma^{n}} \frac{1}{\Omega+i \gamma}, \ldots
$$

which correspond in $t$-representation to the set of Laguerre functions: $\exp (-\gamma t), \ldots t^{n} \exp (-\gamma t) \ldots$ With these functions one may compute analytically all the needed integrals and they have a reasonable asymptotic behavior for large $t$.

The explicit solution of Eqs. (64), (65) is not a trivial task and considerable amount of work will be called for in the future to find and to analyze it. In this paper we proceed on qualitative grounds to demonstrate that the solvability condition of these equations contains the phenomenon of anomalous scaling. For this purposes we will employ just the first non-vanishing term in the set (66) as displayed below:

$$
\begin{aligned}
\tilde{\mathcal{F}}_{2 ; h}(\Omega) & \Rightarrow F \frac{\gamma}{\Omega^{2}+\gamma^{2}}, \\
\mathcal{T}_{A ; h}^{(0)}\left(\Omega_{-}, \Omega_{+}\right) & \Rightarrow T \frac{1}{\left(\Omega_{+}-i \gamma_{1}\right)^{2}\left(\Omega_{-}+i \gamma_{1}\right)^{2}} .
\end{aligned}
$$

One sees that for $\tilde{\mathcal{F}}_{2 ; h}(\Omega)$ we took $\operatorname{Im} f_{0}(\Omega)$ as required by the evenness of a real function $\tilde{\mathcal{F}}_{2 ; h}(\Omega)$. In $t$ representation the triplex $\mathcal{T}_{A ; h}\left(t_{-}, t_{+}\right)$must be zero if $t_{+}$and/or $t_{-}$are less or equal to zero. The homogeneous evolution equations for the triplices dictate that they are small for small $t_{+}$and/or $t_{-}$. Therefore there is no contribution of $f_{0}(\Omega)$ to the triplices, and we employ the representation (68) in which the choice between $f_{1}(\Omega)$ and $f_{1}^{*}(\Omega)$ is dictated by causality. We introduced in Eq. (68) an additional freedom $\left(\gamma_{1} \neq \gamma\right)$ which will allow to see later how qualitative results depend on the choice of the trial functions. The results are only weakly sensitive to the ratio $\gamma_{1} / \gamma$.

As one knows for example from quantum mechanical calculations of energy levels, eigenvalues are usually much less sensitive to the choice of trial functions than the corresponding eigenfunctions. This provides an additional justification to the expectation that our eigenvalue problem of finding $\mathcal{Z}(h)$ from the solvability condition of Eqs. (64), (65) is still meaningful even with very simple trial functions (67), (68). To formulate the solvability condition we consider a consequence of Eq. (65) which may be called their "one-frequency" reduction: multiply Eq. (62) for $\tilde{\mathcal{T}}_{h}^{(0)}$ by $\delta\left(\Omega+\Omega_{-}-\Omega_{+}\right)$and integrate over $\Omega_{+}$and $\Omega_{-}$. This gives:

$$
-\left[i \Omega+\tilde{\sigma}_{h}(\Omega)\right] \tilde{\mathcal{T}}_{h}(\Omega)=\epsilon^{4}\left[\tilde{\mathcal{D}}_{h}(\Omega)+\tilde{\mathcal{E}}_{h}(\Omega)\right],
$$

in which

$$
\begin{aligned}
\tilde{\mathcal{T}}_{h}(\Omega) & \equiv \int \frac{d \Omega_{-} d \Omega_{+} \delta\left(\Omega+\Omega_{-}-\Omega_{+}\right)}{2 \pi} \tilde{\mathcal{T}}_{h}^{(0)}\left(\Omega_{-}, \Omega_{+}\right), \\
\tilde{\mathcal{D}}_{h}(\Omega) & \equiv \int \frac{d \Omega_{-} d \Omega_{+} \delta\left(\Omega+\Omega_{-}-\Omega_{+}\right)}{2 \pi} \tilde{\mathcal{D}}_{h}^{(0)}\left(\Omega_{-}, \Omega_{+}\right), \\
\tilde{\mathcal{E}}_{h}(\Omega) & \equiv \int \frac{d \Omega_{-} d \Omega \delta\left(\Omega+\Omega_{-} \Omega_{+}\right) p}{2 \pi} \tilde{\mathcal{E}}_{h}^{(0)}\left(\Omega_{-}, \Omega_{+}\right) .
\end{aligned}
$$

Equation (69) has exactly the same factor $\left[i \Omega+\tilde{\sigma}_{h}(\Omega)\right]$ in its LHS as Eq. (64) for $\tilde{\mathcal{F}}_{2 ; h}(\Omega)$ :

$$
-\left[i \Omega+\tilde{\sigma}_{h}(\Omega)\right] \tilde{\mathcal{F}}_{2 ; h}(\Omega)=\tilde{\mathcal{B}}_{h}(\Omega) .
$$

This allows the elimination of the non-homogeneous terms $\propto \Omega$, multiplying Eq. (73) by $\mathcal{T}_{h}(\Omega)$ and Eq. (69) by $\tilde{\mathcal{F}}_{2 ; h}(\Omega)$ and taking their difference:

$$
\tilde{\mathcal{T}}_{h}(\Omega) \tilde{\mathcal{B}}_{h}(\Omega)=\epsilon^{4} \tilde{\mathcal{F}}_{2 ; h}(\Omega)\left[\tilde{\mathcal{D}}_{h}(\Omega)+\tilde{\mathcal{E}}_{h}(\Omega)\right] .
$$

Recall that according to (B24) $\tilde{\mathcal{B}}_{h}$ is a form linear in the triplices $\mathcal{T}_{A ; h}$ and quadratic in $\mathcal{F}_{2 ; h}$, while $\tilde{\mathcal{D}}_{h}$ and $\tilde{\mathcal{D}}_{h}$ are forms quadratic in $\tilde{\mathcal{T}}_{A ; h}$ and linear in $\tilde{\mathcal{F}}_{2 ; h}$. Therefore Eq. (74) is a homogeneous form which is quadratic both in $\tilde{\mathcal{T}}_{A ; h}$ and $\tilde{\mathcal{F}}_{2 ; h}$. With our representation (67), (68) of $\mathcal{F}_{2 ; h}$ and $\tilde{\mathcal{T}}_{A ; h}$ Eq. $(74)$ is proportional to $F^{2} T^{2}$ which 
may be canceled if there exists a non-trivial solution of the problem. Therefore one may think that Eq. (74) is the solvability condition which we are looking for. However, the trial functions $(\sqrt[67]{ })$ and $(\sqrt[68)]{ })$ are not a solution of Eqs. (73), (69). Therefore we cannot expect to satisfy Eq. (74) for all values of the frequency. According to our calculational scheme we have to project the initial Eqs. (69), (73) on the (truncated) set of functions $f_{0}(\Omega), f_{1}(\Omega)$ and consider the algebraic consequences of this step. Actually Eq. (74) sheds light on how we may proceed in order to find a solvability condition in our scheme. Namely, we may project Eq. (73) for $\mathcal{F}_{2 ; h}$ on the triplex trial function (68) and Eq. (69) for $\tilde{\mathcal{T}}_{A ; h}$ on the trial function $(67)$ for $\mathcal{F}_{2 ; h}$ and subtract the results. This procedure corresponds to $\Omega$-integration of Eq. (74):

$$
\begin{aligned}
& \int \frac{d \Omega}{2 \pi} \tilde{\mathcal{T}}_{h}(\Omega) \tilde{\mathcal{B}}_{h}(\Omega) \\
= & \epsilon^{4} \int \frac{d \Omega}{2 \pi}\left[\tilde{\mathcal{F}}_{2 ; h}(\Omega) \tilde{\mathcal{D}}_{h}(\Omega)+\tilde{\mathcal{F}}_{2 ; h}(\Omega) \tilde{\mathcal{E}}_{h}(\Omega)\right] .
\end{aligned}
$$

In Eq. (75) $\tilde{\mathcal{B}}_{h}(\Omega)$ is given by Eqs. (B24) $-(\sqrt{\mathrm{B} 27}), \tilde{\mathcal{D}}_{h}(\Omega)$ and $\tilde{\mathcal{E}}_{h}(\Omega)$ are defined by Eqs. (71) and (71) in which $\tilde{\mathcal{D}}_{h}^{(0)}\left(\Omega_{-}, \Omega_{+}\right)$and $\tilde{\mathcal{E}}_{h}^{(0)}\left(\Omega_{-}, \Omega_{+}\right)$are given by (B28) (B30). All these equations contain the functions $\tilde{\mathcal{F}}_{2 ; h}(\Omega)$ and $\tilde{\mathcal{T}}_{h}(\Omega)$ for which we will use the representations (67) and (68). Finally Eq. (75) yields:

$$
\frac{a}{R_{\mathcal{Z}}} U_{a}(h)+b U_{b}(h)+c R_{\mathcal{Z}} U_{c}(h)=0,
$$

where $R_{\mathcal{z}}=\lambda^{2+\mathcal{Z}(h)}$ and $U_{a}(h), U_{b}(h)$ and $U_{c}(h)$ are $\Omega$-integrals of $U_{a ; h}(\Omega), U_{b ; h}(\Omega)$ and $U_{c ; h}(\Omega)$ and

$$
\begin{aligned}
& U_{a ; h}(\Omega) \equiv V_{a ; h}(\Omega)-\epsilon^{4} W_{a ; h}(\Omega), U_{b ; h}(\Omega) \equiv V_{b ; h}(\Omega), \\
& U_{c ; h}(\Omega) \equiv V_{c ; h}(\Omega)-\epsilon^{4} W_{c ; h}(\Omega) .
\end{aligned}
$$

Equation (76) are quadratic with respect to $R_{\mathcal{z}}=$ $\lambda^{2+\mathcal{Z}(h)}$ and allows to find $\mathcal{Z}$ as a function of $h$. The actual calculation has been done with the help of Matematica, a system for doing analytical calculations by a computer [13]. The resulting set of functions $\mathcal{Z}(h)$ for $\epsilon=$ $0,0.25,0.5,0.75,1$, for the spacing parameters $\lambda=2,4$ and the parameter $b=0,-0.5,-1$ is shown in Fig. 10 . Figure 11 displays the functions $\mathcal{Z}(h)$ for the same set of $\epsilon, b=-2$ and $\lambda=2,4,8$. Note that at for $\epsilon=0$ (solid lines) in both Figs.10 and 11 at $h=1$ all the functions $\mathcal{Z}(h)$ are equal to -2 (this is relatively easy to see analytically). For $h<1$ one sees that the functions $\mathcal{Z}(h)$ go down almost as straight lines. An important conclusion is that at $\epsilon=0$ the functions $\mathcal{Z}(h)$ are always negative (moreover, $\mathcal{Z}(h) \leq-2$ ). Recall that the scaling exponents $\zeta_{n}$ are related with $\mathcal{Z}(h)$ via saddle-point requirement (49) and, in particular, $\zeta_{0}=\min _{h} \mathcal{Z}(h)$. By definition, the zero-order structure function is 1 , therefore $\zeta_{0}=0$ and, consequently, for physical solutions $\mathcal{Z}(h) \geq 0$. Negative values of $\mathcal{Z}(h)$ are unphysical like the negative values of masses which may appear as a solution for equilibrium of a mechanical system. Remember that in the derivation of the closure equations we have neglected inhomogeneous terms with normal K41 scaling with respect of terms with anomalous scaling; this is only possible if $\mathcal{Z}(h)>0$. Therefore for negative values of $\mathcal{Z}(h)$ the "anomalous" solutions must be disregarded in favor of solutions with normal scaling. The first important conclusion is therefore that at $\epsilon=0$ the qualitative analysis of the controlled closure predicts K41 scaling.

We next consider the parameter range for which $a c<$ 0 . This mimics the Navier Stokes case in directing the cascade from large to small scales. The parameter $b$ is chosen in the region of stability $-2<b<0$, for which the effect of the flux of the second ("helicity") integral of motion becomes irrelevant deep in the inertial interval. With $a$ taken to be unity, these two constraints limit $b$ to the interval $[-1,0]$. Indeed, in most previous simulations the value $b=-0.5$ was chosen. The calculated functions $\mathcal{Z}(h)$ for this region of $b$ are exhibited in Fig. 10. One sees that in all the panels the values of $\mathcal{Z}(h)$ increase with $\epsilon$ and some of the functions $\mathcal{Z}(h)$ become positive in the left part of the $h$ interval. Moreover, $\mathcal{Z}(h)$ gains a minimum (although still in the negative region). All these qualitative features are precisely those expected for the functions $\mathcal{Z}(h)$ in the case of anomalous scaling. We note that the present level of analysis cannot yield quantitative results. The functions $\mathcal{Z}(h)$ are still mostly negative, and we only see the good trend of creating a minimum and inching up toward positive territory with the increase in $\epsilon$. Although we cannot discuss at present the actual values of the anomalous scaling exponents, their dependence on the parameters of the model: $\epsilon, \lambda$ and $b$, etc, we think that results in the Fig. 10 can be considered as definite evidence for the birth of anomalous scaling with the increase of the value of $\epsilon$. An additional encouraging result is the finding that in the non-physical interval of $b$ (see in Fig. 11) we do not see any of the good phenomena discussed above: at $\epsilon=0$ we have two negative branches of $\mathcal{Z}(h)$ and with increasing of the value of $\epsilon$ the solutions stay in between these branches without a tendency to increase. Moreover for $\epsilon>1 / 2$ the solutions $\mathcal{Z}(h)$ disappear altogether. 

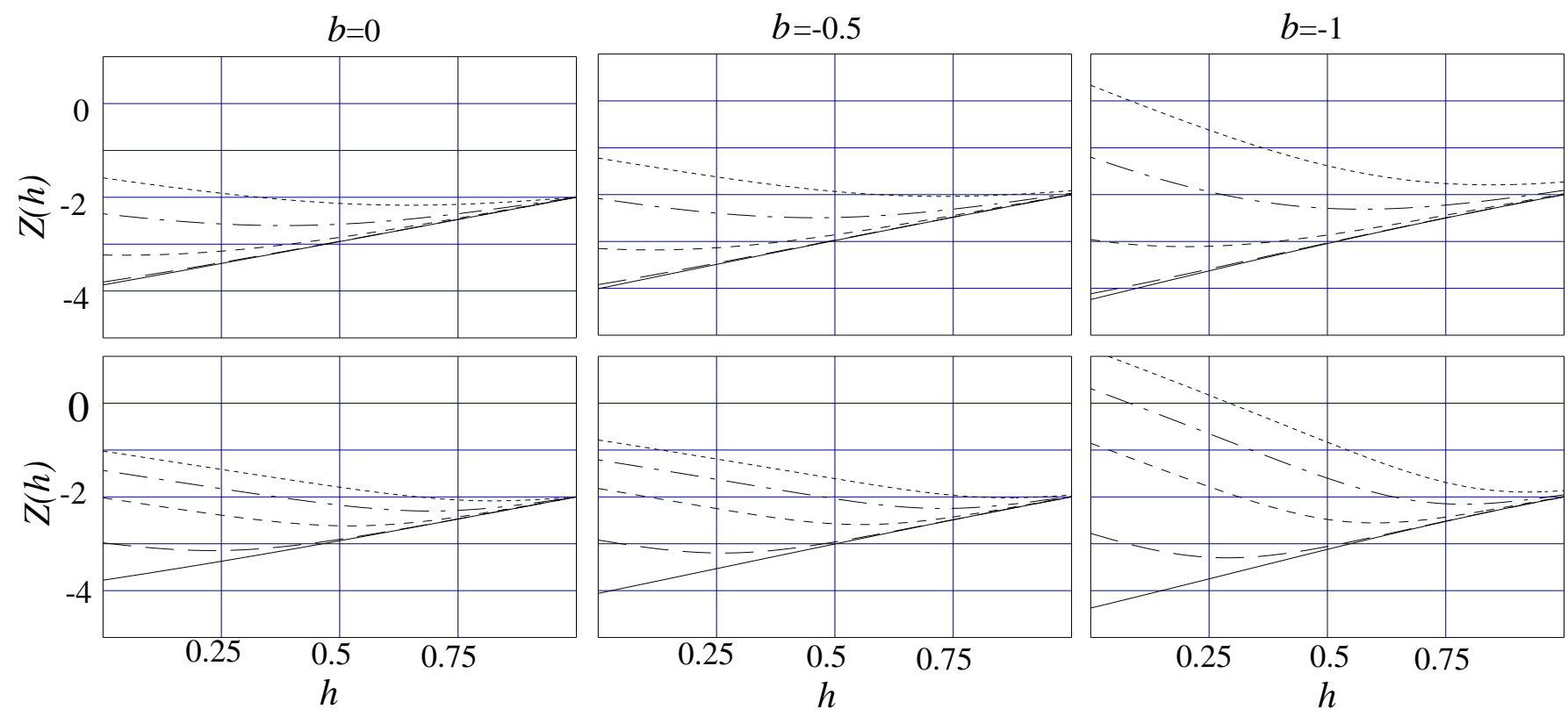

FIG. 10. The function $\mathcal{Z}(h)$ for different values of $\epsilon, b$ and $\lambda$. Different line types denote different values of $\epsilon: \epsilon=0,0.25,0.5$, 0.75 and 1 are shown with solid, long dashed, dashed, dash-dotted and dotted lines respectively. The three columns correspond to $b=0,-0.5$ and -1 , as shown over the panels. The upper panels correspond to $\lambda=2$, the lower ones to $\lambda=4$. The ratio $\gamma_{1} / \gamma=0.5$.
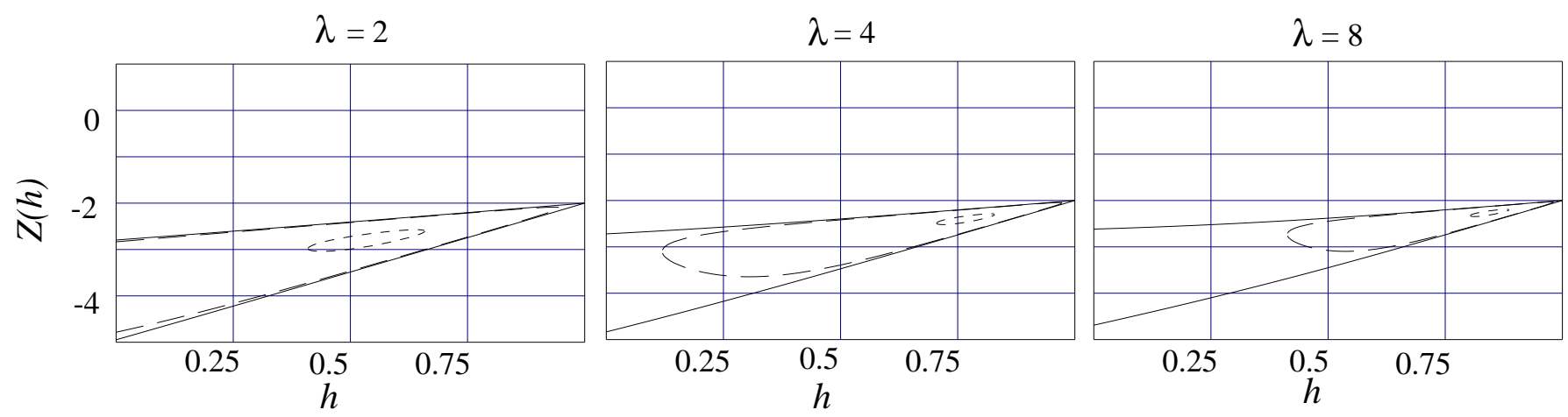

FIG. 11. The functions $\mathcal{Z}(h)$ at $b=-2$ and $\gamma_{1} / \gamma=0.5$. The line types correspond to those in the previous figure. The values of $\lambda$ are shown over the different panels.

\section{SUMMARY AND DISCUSSION}

For the sake of clarity we summarize verbally the important steps in this paper.

1. We first introduced the $(N, \epsilon)$-shell model in which $N$ copies of the "Sabra" shell model are coupled with the interaction amplitudes shown in Eq.(20). These amplitudes contain a deterministic and a random part with the parameter $\epsilon$ determining their relative importance.

2. For $\epsilon=0$ the model reduces to the well known random coupling model [7], in which there is no anomalous scaling in the limit $N \rightarrow \infty$. For $\epsilon=1$ the system boils down to $N$ uncoupled copies of the "Sabra" model with anomalous scaling for each.

3. We studied numerically the behavior of the model for values of $\epsilon$ in the interval $[0,1]$ in the limit $N \rightarrow \infty$. The results are shown in Figs.1 and 2. For $\epsilon=0$ we find no anomalous scaling as expected, for $\epsilon=0.8$ we find anomalous corrections smaller than those found for the Sabra model which pertains to $\epsilon=1$.

4. We derived the linear hierarchy of evolution equations for the correlation and response functions of this model. In the inviscid limit the hierarchy of equations for the correlation functions is linear and 
homogeneous.

5. We pointed out that the homogeneity of the hierarchy of equations results in a rescaling symmetry of these equations which foliates the solutions on socalled $h$-slices, where $h$ is the rescaling exponents of the velocity variable. The $n$th order velocity correlation function rescales on an $h$-slice with a scaling exponent $n h+\mathcal{Z}(h)$, where $\mathcal{Z}(h)$ is associated with the rescaling properties of the probability measure.

6. The full solution is written as an integral over contributions with different $h$, and leads to multiscaling via the saddle point calculation of the corresponding integrals over $h$. The values of the scaling exponents are determined by the function $\mathcal{Z}(h)$ according to Eq.(49).

7. The hierarchical equations written on an $h$ slice can be closed in a controlled fashion using $\epsilon$ as a small parameter. We derived the closed equations for the 2nd and 3rd order objects, retaining terms up to $O\left(\epsilon^{4}\right)$ and showing that the parts neglected are of $O\left(\epsilon^{6}\right)$. We could go a further step in the closure scheme by writing down a system for the $2 \mathrm{nd}$, 3rd and 4 th order objects, retaining terms up to $O\left(\epsilon^{6}\right)$ and neglecting terms of $O\left(\epsilon^{8}\right)$ etc.

8. Section VIII presents a preliminary analysis of $\mathcal{Z}(h)$ from solvability condition for the closed set of equations for the 2nd and 3rd order objects. The full analysis is technically involved and calls for further work. The main qualitative conclusions of the present analysis is that there exists no anomalous solution for $\epsilon=0$, whereas for larger values of $\epsilon$ we see the birth of anomalous scaling. The level of precision is currently not sufficient for quantitative comparisons with the numerical simulations, but we judge the results extremely encouraging for further work in the near future.

In summary, we presented above what appears to be the first attempt to compute from the equations of motion the anomalous exponents of the correlations of a nonlinear turbulent system in a controlled fashion. The small parameter $\epsilon$ has been used to close the infinite hierarchy of equations for the statistical objects, from which one can compute the 2 nd and 3rd correlation functions explcitly. Nevertheless we considered the calculation of $\mathcal{Z}(h)$ only, and also this calculation turned out to be too hard to be performed exactly. The bottom line of this analysis is that (i) the anomaly of the scaling exponents is analytically predictable by the method developed above, and (ii) the actual calculation of numbers is rather difficult and calls for additional careful work.

\section{ACKNOWLEDGMENTS}

We thank Barak Galanti for his assistance with the numerical simulations, and to Gregory Eyink for sharing with us his unpublished results in [9]. The work has been supported in part by the European Commission under the Training and Mobility of Researchers program, The German-Israeli Foundation, the Israel Science Foundation administered by the Israel Academy of Sciences, and the Naftali and Anna Backenroth-Bronicki Fund for Research in Chaos and Complexity.

\section{APPENDIX A: SKETCH OF DERIVATION OF THE HIERARCHY OF EVOLUTION EQUATIONS}

\section{Hierarchy for the correlation functions}

Let us compute $\partial F_{2 ; \sigma, \sigma}^{\alpha}\left(k_{n} ; t-t^{\prime}\right) / \partial t$ defined by Eq. (23) at $t^{\prime}=0$, substituting instead of $d u_{n, \sigma}^{\alpha}(t) / d t$ the RHS of Eq. (19). With the help of definition (24) the result may be written as:

$$
\begin{aligned}
& \frac{\partial}{\partial t} F_{2 ; \sigma, \sigma}^{\alpha}\left(k_{n} ; t\right)=\sum_{\beta, \gamma, \sigma^{\prime}, \sigma^{\prime \prime}} \Phi^{\gamma, \beta, \alpha}\left[\Delta_{\alpha+\beta, \gamma}+\Delta_{\alpha+\beta+N, \gamma}+\Delta_{\alpha+\beta, \gamma+N}\right] A_{\sigma^{\prime} \sigma^{\prime \prime}}^{\sigma} \\
& \times\left\{a k_{n+1} F_{3, \sigma \sigma^{\prime} \sigma^{\prime \prime}}^{\alpha, \beta, \gamma}\left(k_{n+1} ; 0, t, t\right)+b k_{n} F_{3, \sigma \sigma^{\prime} \sigma^{\prime \prime}}^{\alpha, \beta, \gamma}\left(k_{n} ; t, 0, t\right)+c k_{n-1} F_{3, \sigma \sigma^{\prime} \sigma^{\prime \prime}}^{\alpha, \beta, \gamma}\left(k_{n-1} ; t, t, 0\right)\right\},
\end{aligned}
$$

where we used the symmetry properties $A_{\sigma^{\prime} \sigma^{\prime \prime}}^{\sigma}=A_{\sigma \sigma^{\prime \prime}}^{\sigma^{\prime}}$. Summing this equation over $\alpha$ and $\sigma$ we derive Eqs. (36) with the invariant combination (34) in the RHS.

\section{Definitions of the Green's function}

aa:defG

First we define the "tensorial" Green's function 


$$
G_{1,1 ; \sigma, \sigma^{\prime}}^{\alpha, \beta}\left(k_{n} ; t_{1}-t_{2}\right)=\left\langle\frac{\delta u_{n, \sigma}^{\alpha}\left(t_{1}\right)}{\delta f_{n, \sigma^{\prime}}^{\beta *}\left(t_{2}\right)}\right\rangle,
$$

and its scalar counterpart which is invariant under all the symmetry transformations described in Subsect. IV A:

$$
G_{1,1}\left(k_{n} ; t_{1}-t_{2}\right) \equiv \frac{1}{N} \sum_{\alpha, \sigma} G_{1,1 ; \sigma, \sigma}^{\alpha, \alpha}\left(k_{n} ; t_{1}-t_{2}\right) .
$$

Next we define the 3 rd order tensorial Green's functions $\boldsymbol{G}_{2,1}$ and $\boldsymbol{G}_{1,2}$ describing a response of two velocities on one forcing and vice versa:

$$
\begin{aligned}
& G_{2,1 ; \sigma \sigma^{\prime} \sigma^{\prime \prime}}^{\alpha, \beta, \gamma}\left(k_{n}, k_{m}, k_{\ell} ; t_{n} ; t_{m}, t_{\ell}\right) \equiv\left\langle\frac{\delta\left[u_{m, \sigma^{\prime}}^{\beta}\left(t_{m}\right) u_{l, \sigma^{\prime \prime}}^{\gamma}\left(t_{\ell}\right)\right]}{\delta f_{n, \sigma}^{\alpha *}\left(t_{n}\right)}\right\rangle \\
& G_{1,2 ; \sigma \sigma^{\prime} \sigma^{\prime \prime}}^{\alpha, \beta, \gamma}\left(k_{n}, k_{m}, k_{\ell} ; t_{n}, t_{m}, t_{\ell}\right) \equiv\left\langle\frac{\delta^{2} u_{l, \sigma^{\prime \prime}}^{\gamma}\left(t_{\ell}\right)}{\left[\delta f_{m, \sigma^{\prime}}^{\beta}\left(t_{m}\right) \delta f_{n, \sigma}^{\alpha}\left(t_{n}\right)\right]^{*}}\right\rangle .
\end{aligned}
$$

Analogously to the definition of the scalar 3rd order correlation function (34) we define here the scalar counterparts of these 3rd order Green's functions:

$$
\begin{aligned}
G_{2,1}\left(k_{n}, k_{m}, k_{\ell} ; t_{n} ; t_{m}, t_{\ell}\right) \equiv & \frac{1}{N} \sum_{a, \beta, \gamma} \sum_{\sigma, \sigma^{\prime} \sigma^{\prime \prime}} \Phi^{\gamma, \beta, \alpha} A_{\sigma^{\prime} \sigma^{\prime \prime}}^{\sigma}\left[\Delta_{\alpha, \beta+\gamma}+\Delta_{\alpha+N, \beta+\gamma}+\Delta_{\alpha, \beta+\gamma+N}\right] \\
& \times G_{2,1, \beta, \gamma \sigma^{\prime} \sigma^{\prime \prime}}^{\alpha,}\left(k_{n}, k_{m}, k_{\ell} ; t_{n} ; t_{m}, t_{\ell}\right), \\
G_{1,2}\left(k_{n}, k_{m}, k_{\ell} ; t_{n} ; t_{m}, t_{\ell}\right) \equiv & \frac{1}{N} \sum_{a, \beta, \gamma \sigma, \sigma^{\prime} \sigma^{\prime \prime}} \sum^{\gamma, \beta, \alpha} A_{\sigma^{\prime} \sigma^{\prime \prime}}^{\sigma}\left[\Delta_{\alpha, \beta+\gamma}+\Delta_{\alpha+N, \beta+\gamma}+\Delta_{\alpha, \beta+\gamma+N}\right] \\
& \times G_{1,2 ; \sigma \sigma^{\prime} \sigma^{\prime \prime}}^{\alpha, \beta}\left(k_{n}, k_{m}, k_{\ell} ; t_{n}, t_{m}, t_{\ell}\right) .
\end{aligned}
$$

As we discussed in Subsect. VIA any three point objects of the Sabra-shell model differ from zero only for three consecutive shell numbers. This allows one to consider instead of each Green's function (A7) and (A6) of three $k$ arguments three functions with just one (middle) $k$ argument. Exactly like in the case of triplices we introduce superscripts ${ }^{(0)}$ and ${ }^{( \pm)}$to distinguish them. Using the convention discussed in Subsect. VIA we can define $G_{2,1}^{(0)}$, $G_{2,1}^{( \pm)}, G_{2,1}^{(0)}$ and $G_{2,1}^{( \pm)}$. For example:

$$
G_{2,1}^{(0)}\left(k_{m} ; t_{m-1} ; t_{m}, t_{m+1}\right) \equiv G_{2,1}\left(k_{m}, k_{m-1}, k_{m+1} ; t_{m} ; t_{m-1}, t_{m+1}\right) .
$$

Similarly we define the successive scalar response functions of the 4th order as follows:

$$
\begin{aligned}
& G_{3,1}\left(k_{n 1}, k_{n 2}, k_{n 3}, k_{n 4} ; t_{1}, t_{2}, t_{3}, t_{4}\right) \equiv \frac{1}{N^{2}} A_{\sigma^{\prime \prime} \sigma_{3}}^{\sigma^{\prime}} A_{\sigma_{4} \sigma_{5}}^{\sigma^{\prime}}\left\langle\frac{\delta\left(u_{n 1, \sigma^{\prime \prime}}^{\beta *}\left(t_{2}\right) u_{n 2, \sigma_{3}}^{\alpha}\left(t_{3}\right) u_{n 3, \sigma_{4}}^{\alpha *}\left(t_{4}\right)\right)}{\delta f_{n 1, \sigma^{\prime}}^{\beta}\left(t_{1}\right)}\right\rangle, \\
& G_{2,2}\left(k_{n 1}, k_{n 2}, k_{n 3}, k_{n 4} ; t_{1}, t_{2}, t_{3}, t_{4}\right) \equiv \frac{1}{N^{2}} A_{\sigma^{\prime \prime} \sigma_{3}}^{\sigma^{\prime}} A_{\sigma_{4} \sigma_{5}}^{\sigma^{\prime}}\left\langle\frac{\delta\left(u_{n 2, \sigma^{\prime \prime}}^{\beta *}\left(t_{2}\right) u_{n 3, \sigma_{3}}^{\alpha}\left(t_{3}\right)\right)}{\delta f_{n 1, \sigma^{\prime}}^{\beta}\left(t_{1}\right) \delta f_{n 4, \sigma_{4}}^{\alpha *}\left(t_{4}\right)}\right\rangle, \cdots
\end{aligned}
$$

with the convention of sums over repeated indices. Note that all the $G$ 's are real functions. Again we note that it is more convenient to write the hierarchical equations in the terms of quantities summed over $\sigma$ 's. These are invariant under all the symmetry transformations discussed in Sect. IV A.

\section{Hierarchical equations for the Green's functions}

The tensorial equation for the 2 nd order $G_{1,1 ; \sigma \sigma}^{\alpha, \alpha}$ reads:

$$
\begin{aligned}
\frac{\partial}{\partial t} G_{1,1 ; \sigma, \sigma}^{\alpha, \alpha}\left(k_{n} ; t\right)= & \sum_{\beta, \gamma, \sigma^{\prime}, \sigma^{\prime \prime}} \Phi^{\gamma, \beta, \alpha}\left[\Delta_{\alpha+\beta, \gamma}+\Delta_{\alpha+\beta+N, \gamma}+\Delta_{\alpha+\beta, \gamma+N}\right]\left\{A _ { \sigma ^ { \prime } \sigma ^ { \prime \prime } } ^ { \sigma } \left[\gamma_{a, n+1} G_{2,1 ; \sigma \sigma^{\prime} \sigma^{\prime \prime}}^{\alpha, \beta, \gamma}\left(k_{n}, k_{n+1}, k_{n+2} ; 0, t, t\right)\right.\right. \\
& \left.\left.+\gamma_{b, n} G_{2,1 ; \sigma \sigma^{\prime} \sigma^{\prime \prime}}^{\alpha, \beta, \gamma}\left(k_{n}, k_{n-1}, k_{n+1} ; 0, t, t\right)\right]+C_{\sigma^{\prime} \sigma^{\prime \prime}}^{\sigma} \gamma_{c, n-1} G_{2,1 ; \sigma \sigma^{\prime} \sigma^{\prime \prime}}^{\alpha^{\prime}, \beta, \gamma}\left(k_{n}, k_{n-2}, k_{n-1} ; 0, t, t\right)\right\}+\delta(t) .
\end{aligned}
$$


By following more or less the same procedure as for the correlation functions in Appendix A1 we get the hierarchical equations:

$$
\frac{\partial}{\partial t} G_{1,1}\left(k_{n} ; t\right)=\gamma_{a, n+1} G_{2,1}^{(-1)}\left(k_{n+1} ; 0, t, t\right) \gamma_{b, n} G_{2,1}^{(0)}\left(k_{n} ; t, 0, t\right)+\gamma_{c, n-1} G_{2,1}^{(+1)}\left(k_{n-1} ; t, t, 0\right)+\delta(t) .
$$

Analogously for the higher order Green's functions one has:

$$
\begin{aligned}
\frac{\partial}{\partial t_{1}} G_{2,1}^{(0)}\left(k_{n} ; t_{1}, t_{2}, t_{3}\right)= & \gamma_{a, n} G_{3,1}\left(k_{n}, k_{n}, k_{n+1} k_{n+1} ; t_{2}, t_{1}, t_{1}, t_{3}\right)+\gamma_{b, n-1} G_{3,1}\left(k_{n}, k_{n-2}, k_{n} k_{n+1} ; t_{2}, t_{1}, t_{1}, t_{3}\right) \\
& +\gamma_{c, n-2} G_{3,1}\left(k_{n}, k_{n-3}, k_{n-2} k_{n+1} ; t_{2}, t_{1}, t_{1}, t_{3}\right) \\
\frac{\partial}{\partial t_{1}} G_{1,2}^{(-1)}\left(k_{n} ; t_{1}, t_{2}, t_{3}\right)= & \gamma_{a, n} G_{2,2}\left(k_{n}, k_{n+1}, k_{n}, k_{n+1} ; t_{1}, t_{1}, t_{2}, t_{3}\right)+\gamma_{b, n-1} G_{2,2}\left(k_{n-2}, k_{n}, k_{n} k_{n+1} ; t_{1}, t_{1}, t_{2}, t_{3}\right) \\
& +\gamma_{c, n-2} G_{2,2}\left(k_{n-3}, k_{n-2}, k_{n}, k_{n+1} ; t_{1}, t_{1}, t_{2}, t_{3}\right),
\end{aligned}
$$

and similarly for the other $G_{2,1}$ 's and $G_{1,2}$ 's.

\section{APPENDIX B: THE CLOSURE EQUATIONS IN THE $A$-TRIPLEX APPROXIMATION}

\section{Equations in the $\omega$-representation}

Given a stationary case, when all the functions depend on time differences only, it is useful to consider the closure equations in the $\omega$-representation. Define:

$$
\begin{aligned}
& \tilde{F}_{2 ; h}\left(k_{n} ; \omega\right)=\int_{-\infty}^{\infty} F_{2 ; h}\left(k_{n} ; t\right) \exp (i \omega t) d t \\
& \tilde{F}_{2 ; h}^{*}\left(k_{n} ; \omega\right)=\int_{-\infty}^{\infty} F_{2 ; h}^{*}\left(k_{n} ; t\right) \exp (-i \omega t) d t .
\end{aligned}
$$

As in these equations, we will use tilde signs over a character to denote a Fourier transform of that function. It follows from Eq. (B1) that

$$
F_{2}\left(k_{n} ; t\right)=\int_{-\infty}^{\infty} \tilde{F}_{2}\left(k_{n} ; \omega\right) \exp (-i \omega t) \frac{d \omega}{2 \pi} .
$$

According to the definition 23, 33) $F_{2 ; h}\left(k_{n} ; t\right)=$ $F_{2 ; h}^{*}\left(k_{n} ;-t\right)$. Therefore $\tilde{F}_{2 ; h}\left(k_{n} ; \omega\right)$ is a real function of $\omega$.

Next we define the Fourier transform $\tilde{T}_{h}$ of any $3 r d$ order object $T_{h}$ as follows:

$$
\begin{aligned}
& 2 \pi \tilde{T}_{h}\left(k_{n} ; \omega_{-}, \omega_{0}, \omega_{+}\right) \delta\left(\omega_{-}+\omega_{0}-\omega_{+}\right)=\int_{-\infty}^{\infty} d t_{-} d t_{0} d t_{+} \\
& \times T_{h}\left(k_{n} ; t_{-}, t_{0}, t_{+}\right) \exp \left[i\left(\omega_{-} t_{-}+\omega_{0} t_{0}-\omega_{+} t_{+}\right)\right] . \quad(\mathrm{B} 4)
\end{aligned}
$$

Recall that with our convention (see Subsect. VIA) $t_{0}$ and $t_{ \pm}$are times of the $n$ - and $(n \pm 1)$-shells. With this definition the sum of incoming frequencies $\omega_{-}+\omega_{0}$ is equal to the outgoing frequency $\omega_{+}$.

The equations for the 2nd order correlation function (two top lines in Fig. 5) and for triplices (two last lines) in the $\omega$-representation may be written as:

$$
\begin{aligned}
-\left[i \omega+\tilde{\Sigma}_{h}\left(k_{n} ; \omega\right)\right] \tilde{F}_{2 ; h}\left(k_{n} ; \omega\right) & =\tilde{B}_{h}\left(k_{n}, \omega\right), \\
{\left[i \omega_{+}+\tilde{\Sigma}_{h}^{*}\left(k_{n} ; \omega_{+}\right)\right] \tilde{T}_{h}^{(+1)}\left(k_{n-1} ; \omega_{-}, \omega_{0}\right) } & =\epsilon^{4}\left[\tilde{D}_{h}^{(+1)}\left(k_{n-1} ; \omega_{-}, \omega_{0}\right)+\tilde{E}_{h}^{(+1)}\left(k_{n-1} ; \omega_{-}, \omega_{0}\right)\right], \\
-\left[i \omega_{0}+\tilde{\Sigma}_{h}\left(k_{n} ; \omega_{0}\right)\right] \tilde{T}_{A ; h}^{(0)}\left(k_{n} ; \omega_{-}, \omega_{+}\right) & =\epsilon^{4}\left[\tilde{E}_{h}^{(0)}\left(k_{n}, \omega_{-}, \omega_{+}\right)+\tilde{D}_{h}^{(0)}\left(k_{n}, \omega_{-}, \omega_{+}\right)\right], \\
-\left[i \omega_{-}+\tilde{\Sigma}_{h}\left(k_{n} ; \omega_{-}\right)\right] \tilde{T}_{A ; h}^{(-1)}\left(k_{n+1} ; \omega_{0}, \omega_{+}\right) & =\epsilon^{4}\left[\tilde{D}_{h}^{(-1)}\left(k_{n+1} ; \omega_{0}, \omega_{+}\right)+\tilde{E}_{h}^{(-1)}\left(k_{n+1} ; \omega_{0}, \omega_{+}\right)\right] .
\end{aligned}
$$

Note that the parameter $\epsilon$ appears explicitly here, as it was discussed in Sect.VII. Here the "mass-operator" $\tilde{\Sigma}_{h}\left(k_{n} ; \omega\right)$ originates from the diagram A in Fig. 5 and may be written as:

$$
\begin{aligned}
& \tilde{\Sigma}_{h}\left(k_{n} ; \omega\right)=\frac{1}{2 \pi} \int_{-\infty}^{\infty} d \omega_{-} d \omega_{0} d \omega_{+} \delta\left(\omega_{-}+\omega_{0}-\omega_{+}\right) \\
& \times\left\{\gamma_{n+1,-1}\left[\tilde{F}_{2 ; h}\left(k_{n+2} ; \omega_{+}\right) \tilde{T}_{A ; h}^{(0) *}\left(k_{n+1} ; \omega_{-}, \omega_{+}\right)+\tilde{F}_{2 ; h}\left(k_{n+1} ; \omega_{0}\right) \tilde{T}_{A ; h}^{(+1) *}\left(k_{n+1} ; \omega_{-}, \omega_{0}\right)\right] \delta\left(\omega-\omega_{-}\right)\right.
\end{aligned}
$$




$$
\begin{aligned}
& +\gamma_{n, 0}\left[\tilde{F}_{2 ; h}\left(k_{n+1} ; \omega_{+}\right) \tilde{T}_{A ; h}^{(-1) *}\left(k_{n} ; \omega_{0}, \omega_{+}\right)+\tilde{F}_{2 ; h}\left(k_{n-1} ; \omega_{-}\right) \tilde{T}_{A ; h}^{(+1) *}\left(k_{n} ; \omega_{-}, \omega_{0}\right)\right] \delta\left(\omega-\omega_{0}\right) \\
& \left.+\gamma_{n-1,1}^{*}\left[\tilde{F}_{2 ; h}\left(k_{n-1} ; \omega_{0}\right) \tilde{T}_{A ; h}^{(-1)}\left(k_{n-1} ; \omega_{0}, \omega_{+}\right)+\tilde{F}_{2 ; h}\left(k_{n-2} ; \omega_{-}\right) \tilde{T}_{A ; h}^{(0)}\left(k_{n-1} ; \omega_{-}, \omega_{+}\right)\right] \delta\left(\omega-\omega_{+}\right)\right\} .
\end{aligned}
$$

Here and below we omit, for the sake of brevity, the frequency argument of the leg with the Green's function in $A$ triplices and the corresponding argument in $D$ and $E$ functions. It may be found from the relationship $\omega_{+}=\omega_{-}+\omega_{0}$. The term $\tilde{B}_{h}\left(k_{n}, \omega\right)$ in the RHS of Eq. (B⿹ $)$ corresponds to the diagram B in Fig. 5 ;

$$
\begin{aligned}
\tilde{B}\left(k_{n} ; \omega\right)= & \frac{1}{2 \pi} \int_{-\infty}^{\infty} d \omega_{-} d \omega_{0} d \omega_{+}\left\{\gamma_{n+1,-1} \tilde{F}_{2 ; h}\left(k_{n+1} ; \omega_{0}\right) \tilde{F}_{2 ; h}\left(k_{n+2} ; \omega_{+}\right) \tilde{T}_{A ; h}^{(-1) *}\left(k_{n+1} ; \omega_{0}, \omega_{+}\right) \delta\left(\omega-\omega_{-}\right)\right. \\
& +\gamma_{n, 0} \tilde{F}_{2 ; h}\left(k_{n-1} ; \omega_{-}\right) \tilde{F}_{2 ; h}\left(k_{n+1} ; \omega_{+}\right) \tilde{T}_{A ; h}^{(0) *}\left(k_{n} ; \omega_{-}, \omega_{+}\right) \delta\left(\omega-\omega_{0}\right) \\
& \left.+\gamma_{n-1,1}^{*} \tilde{F}_{2 ; h}\left(k_{n-2} ; \omega_{-}\right) \tilde{F}_{2 ; h}\left(k_{n-1} ; \omega_{0}\right) \tilde{T}_{A ; h}^{(+1)}\left(k_{n-1} ; \omega_{-}, \omega_{0}\right) \delta\left(\omega-\omega_{+}\right)\right\} \delta\left(\omega_{-}+\omega_{0}-\omega_{+}\right) .
\end{aligned}
$$

Functions $D$ correspond to the diagram D:

$$
\begin{aligned}
\tilde{D}_{h}^{(+1)}\left(k_{n-1} ; \omega_{-}, \omega_{0}\right)= & \int_{-\infty}^{\infty} \frac{d \omega}{2 \pi}\left\{\gamma_{n, 0}^{*} \tilde{T}_{A ; h}^{(+1)}\left(k_{n} ; \omega_{0}, \omega\right) \tilde{T}_{A ; h}^{(0)}\left(k_{n-1} ; \omega_{-}, \omega\right) \tilde{F}_{2 ; h}\left(k_{n} ; \omega\right)\right. \\
& \left.+\gamma_{n-1,1} \tilde{T}_{A ; h}^{(0) *}\left(k_{n-2} ; \omega, \omega_{0}\right) \tilde{T}_{A ; h}^{+1}\left(k_{n-2} ; \omega, \omega_{-}\right) \tilde{F}_{2 ; h}\left(k_{n-3} ; \omega\right)\right\}, \\
\tilde{D}_{h}^{(0)}\left(k_{n} ; \omega_{-}, \omega_{+}\right)= & \int_{-\infty}^{\infty} \frac{d \omega}{2 \pi}\left\{\gamma_{n+1,-1} \tilde{T}_{A ; h}^{+1 *}\left(k_{n+1} ; \omega, \omega_{+}\right) \tilde{T}_{1, h}\left(k_{n} ; \omega_{-}, \omega\right) \tilde{F}_{2 ; h}\left(k_{n} ; \omega\right)\right. \\
& \left.+\gamma_{n-1,1}^{*} \tilde{T}_{A ; h}^{(+1)}\left(k_{n} ; \omega, \omega_{+}\right) \tilde{T}_{A ; h}^{(+1)}\left(k_{n-1} ; \omega_{-}, \omega\right) \tilde{F}_{2 ; h}\left(k_{n} ; \omega\right)\right\}, \\
& \int_{-\infty}^{\infty} \frac{d \omega}{2 \pi}\left\{\gamma_{n, 0} \tilde{T}_{A ; h}^{(0)}\left(k_{n+1} ; \omega, \omega_{+}\right) \tilde{T}_{A ; h}^{(-1) *}\left(k_{n} ; \omega, \omega_{0}\right) \tilde{F}_{2 ; h}\left(k_{n} ; \omega\right)\right. \\
& \left.+\gamma_{n+1,-1} \tilde{T}_{A ; h}^{(-1) *}\left(k_{n+2} ; \omega_{+}, \omega\right) \tilde{T}_{A ; h}^{(0)}\left(k_{n+2} ; \omega_{0}, \omega\right) \tilde{F}_{2 ; h}\left(k_{n+3} ; \omega\right)\right\} .
\end{aligned}
$$

Functions $E$ correspond to the diagrams $\mathrm{E} \alpha$ and $\mathrm{E} \beta$ in Fig. 5 :

$$
\begin{aligned}
\tilde{E}_{h}^{(+1)}\left(k_{n-1} ; \omega_{-}, \omega_{0}\right)= & \int_{-\infty}^{\infty} \frac{d \omega}{2 \pi}\left\{\gamma_{n, 0}^{*} \tilde{F}_{2 ; h}\left(k_{n+1} ; \omega\right) \tilde{T}_{A ; h}^{(0)}\left(k_{n-1} ; \omega_{-}, \omega-\omega_{0}\right) \tilde{T}_{A ; h}^{(0)}\left(k_{n} ; \omega_{0}, \omega\right)\right. \\
& +\gamma_{n-1,1} \tilde{F}_{2 ; h}\left(k_{n-2} ; \omega\right) \tilde{T}_{A ; h}^{(+1)}\left(k_{n-2} ; \omega_{0}-\omega, \omega\right) \tilde{T}_{A ; h}^{(-1) *}\left(k_{n-2} ; \omega, \omega_{0}\right) \\
& +\gamma_{n, 0}^{*} \tilde{F}_{2 ; h}\left(k_{n-1} ; \omega\right) \tilde{T}_{A ; h}^{(+1)}\left(k_{n} ; \omega_{0}, \omega+\omega_{-}\right) \tilde{T}_{A ; h}^{(+1)}\left(k_{n-1} ; \omega_{-}, \omega\right) \\
& \left.+\gamma_{n-1,1} \tilde{F}_{2 ; h}\left(k_{n-1} ; \omega\right) \tilde{T}_{A ; h}^{(0) *}\left(k_{n-2} ; \omega-\omega_{-}, \omega_{0}\right) \tilde{T}_{A ; h}^{(+1)}\left(k_{n-2} ; \omega_{-}, \omega\right)\right\}, \\
\tilde{E}_{0}\left(k_{n} ; \omega_{-}, \omega_{+}\right)= & \int_{-\infty}^{\infty} \frac{d \omega}{2 \pi}\left\{\gamma_{n+1,-1} \tilde{F}_{2 ; h}\left(k_{n+2} ; \omega\right) \tilde{T}_{A ; h}^{(+1)}\left(k_{n} ; \omega_{-}, \omega-\omega_{+}\right) \tilde{T}_{A ; h}^{(-1) *}\left(k_{n+1} ; \omega_{+}, \omega\right)\right. \\
& +\gamma_{n-1,1}^{*} \tilde{F}_{2 ; h}\left(k_{n-1} ; \omega\right) \tilde{T}_{A ; h}^{(+1)}\left(k_{n-1} ; \omega_{-}, \omega_{+}-\omega\right) \tilde{T}_{A ; h}^{(0)}\left(k_{n} ; \omega, \omega_{+}\right) \\
& +\gamma_{n+1,-1} \tilde{F}_{2 ; h}\left(k_{n+1} ; \omega\right) \tilde{T}_{A ; h}^{(+1) *}\left(k_{n+1} ; \omega-\omega_{-}, \omega_{+}\right) \tilde{T}_{A ; h}^{(0)}\left(k_{n} ; \omega-, \omega\right) \\
& \left.+\gamma_{n-1,1}^{*} \tilde{F}_{2 ; h}\left(k_{n-2} ; \omega\right) \tilde{T}_{A ; h}^{(+1)}\left(k_{n} ; \omega+\omega_{-}, \omega_{+}\right) \tilde{T}_{A ; h}^{(+1)}\left(k_{n-1} ; \omega, \omega-\right)\right\}, \\
& \int^{\infty} \frac{d \omega}{2 \pi}\left\{\gamma_{n, 0} \tilde{F}_{2 ; h}\left(k_{n+1} ; \omega\right) \tilde{T}_{A ; h}^{(-1) *}\left(k_{n} ; \omega_{+}-\omega, \omega_{0}\right) \tilde{T}_{A ; h}^{(+1)}\left(k_{n+1} ; \omega, \omega_{+}\right)\right. \\
& +\infty \\
\tilde{E}_{A ; h}^{(+1)}\left(k_{n+1} ; \omega_{0}, \omega_{+}\right)= & \gamma_{n+1,-1} \tilde{F}_{2 ; h}\left(k_{n+1} ; \omega\right) \tilde{T}_{A ; h}^{(0)}\left(k_{n+2} ; \omega_{0}, \omega+\omega_{+}\right) \tilde{T}_{A ; h}^{(+1) *}\left(k_{n+2} ; \omega, \omega\right)
\end{aligned}
$$




$$
\begin{aligned}
& +\gamma_{n, 0} \tilde{F}_{2 ; h}\left(k_{n-1} ; \omega\right) \tilde{T}_{A ; h}^{(0)}\left(k_{n+1} ; \omega_{0}-\omega, \omega_{+}\right) \tilde{T}_{A ; h}^{(0) *}\left(k_{n} ; \omega_{4}, \omega_{0}\right) \\
& \left.+\gamma_{n+1,-1} \tilde{F}_{2 ; h}\left(k_{n+2} ; \omega\right) \tilde{T}_{A ; h}^{(-1) *}\left(k_{n+2} ; \omega_{+}, \omega_{0}+\omega\right) \tilde{T}_{A ; h}^{(+1)}\left(k_{n+2} ; \omega_{0}, \omega\right)\right\} .
\end{aligned}
$$

\section{Closure equations for Scale-invariant Functions}

All the objects appearing in the previous equations (B5)-( We make use of this invariance to simplify the closed set of equations by introducing the following scale invariant dimensionless representation:

$$
\begin{aligned}
\tilde{F}_{2 ; h}\left(k_{n} ; \omega\right) & \equiv \frac{U}{k_{0}} \lambda^{-(h+\mathcal{Z}(h)+1)} \tilde{\mathcal{F}}_{2 ; h}\left(\frac{\omega}{\omega_{n ; h}}\right), \quad \tilde{\Sigma}_{h}\left(k_{n} ; \omega\right)=\omega_{n ; h} \tilde{\sigma}_{h}\left(\frac{\omega}{\omega_{n ; h}}\right), \\
\tilde{B}_{h}\left(k_{n} ; \omega\right) & \equiv U^{2} \lambda^{n(1-h)-(h+\mathcal{Z}(h)+1)} \tilde{\mathcal{B}}_{h}\left(\frac{\omega}{\omega_{n ; h}}\right) \\
\tilde{T}_{A ; h}^{(\ell)}\left(k_{n} ; \omega_{a}, \omega_{b}\right) & \equiv \frac{i}{U} \lambda^{n[h+\mathcal{Z}(h)]-\ell[2 h+\mathcal{Z}(h)]} \tilde{\mathcal{T}}_{A ; h}^{(\ell)}\left(\frac{\omega_{a}}{\omega_{n ; h}}, \frac{\omega_{b}}{\omega_{n ; h}}\right), \quad \ell=-1,0,1, \\
\tilde{D}_{A ; h}^{(\ell)}\left(k_{n} ; \omega_{a}, \omega_{b}\right) & \equiv i k_{0} \lambda^{n[1+\mathcal{Z}(h)]-\ell[2 h+\mathcal{Z}(h)]} \tilde{\mathcal{D}}_{A ; h}^{(\ell)}\left(\frac{\omega_{a}}{\omega_{n ; h}}, \frac{\omega_{b}}{\omega_{n ; h}}\right), \\
\tilde{E}_{A ; h}^{(\ell)}\left(k_{n} ; \omega_{a}, \omega_{b}\right) & \equiv i k_{0} \lambda^{n[1+\mathcal{Z}(h)]-\ell[2 h+\mathcal{Z}(h)]} \tilde{\mathcal{E}}_{A ; h}^{(\ell)}\left(\frac{\omega_{a}}{\omega_{n ; h}}, \frac{\omega_{b}}{\omega_{n ; h}}\right),
\end{aligned}
$$

Here the characteristic frequency of $n$-shell on an $h$-slice is

$$
\omega_{n ; h} \equiv U k_{0} \mu^{n}, \quad \mu \equiv \lambda^{1-h},
$$

$\lambda$ is a spacing parameter in the shell models determined as the ratio between two consecutive shell momenta: $\lambda=$ $k_{n+1} / k_{n}$. Recall that the dependence on a single $k_{n}$ is represents in the case of 3rd order functions a dependence on three consecutive shell momenta $k_{n-1}, k_{n}$ and $k_{n+1}$. The superscript ${ }^{(\ell)}$ determines the $k$-argument of the "special leg" accordingly to the convention of Sect. VIA.

In the dimensionless form Eqs. (B9) are scale invariant, independent of the shell number. In these Eqs.:

$$
\begin{aligned}
& \mathcal{B}_{h}(\Omega)=\frac{a}{R_{\mathcal{Z}}} V_{a ; h}(\Omega)+b V_{b ; h}(\Omega)+c R_{\mathcal{z}} V_{c ; h}(\Omega), \quad \text { where } \quad R_{\mathcal{Z}} \equiv \lambda^{2+\mathcal{Z}(h)}, \\
& V_{a ; h}(\Omega)=\int \frac{d \Omega_{1} d \Omega_{2} \delta\left(\Omega+\Omega_{1}-\Omega_{2}\right)}{2 \pi} \tilde{\mathcal{F}}_{2 ; h}\left(\frac{\Omega_{1}}{\mu}\right) \tilde{\mathcal{F}}_{2 ; h}\left(\frac{\Omega_{2}}{\mu^{2}}\right) \tilde{\mathcal{T}}_{A ; h}^{(-1)}\left(\frac{\Omega_{1}}{\mu}, \frac{\Omega_{2}}{\mu}\right), \\
& V_{b ; h}(\Omega)=\int \frac{d \Omega_{1} d \Omega_{2} \delta\left(\Omega+\Omega_{1}-\Omega_{2}\right)}{2 \pi} \tilde{\mathcal{F}}_{2 ; h}^{*}\left(\Omega_{1} \mu\right) \tilde{\mathcal{F}}_{2 ; h}\left(\frac{\Omega_{2}}{\mu}\right) \tilde{\mathcal{T}}_{A ; h}^{(0) *}\left(\Omega_{1}, \Omega_{2}\right), \\
& V_{c ; h}(\Omega)=\int \frac{d \Omega_{1} d \Omega_{2} \delta\left(\Omega-\Omega_{1}-\Omega_{2}\right)}{2 \pi} \tilde{\mathcal{F}}_{2 ; h}\left(\Omega_{1} \mu\right) \tilde{\mathcal{F}}_{2 ; h}\left(\Omega_{2} \mu^{2}\right) \tilde{\mathcal{T}}_{A ; h}^{(+1)}\left(\Omega_{2} \mu, \Omega_{1} \mu\right)
\end{aligned}
$$

The RHS of Eq. (62) for $\mathcal{T}_{A ; h}^{(0)}$ has the form:

$$
\begin{aligned}
& \tilde{\mathcal{E}}_{h}^{(0)}\left(\Omega_{-}, \Omega_{+}\right)+\tilde{\mathcal{D}}_{h}^{(0)}\left(\Omega_{-}, \Omega_{+}\right)=\frac{a}{R_{z}} W_{a ; h}\left(\Omega_{-}, \Omega_{+}\right)+c R_{z} W_{c ; h}\left(\Omega_{-}, \Omega_{+}\right), \quad \text { where } \\
& W_{a ; h}\left(\Omega_{-}, \Omega_{+}\right)=\int \frac{d \Omega}{2 \pi}\left\{\mu^{3} \tilde{\mathcal{F}}_{2 ; h}(\Omega) \tilde{\mathcal{T}}_{A ; h}^{(+1) *}\left(\frac{\Omega}{\mu}, \frac{\Omega_{+}}{\mu}\right) \tilde{\mathcal{T}}_{A ; h}^{(+1)}\left(\Omega_{-}, \Omega\right)\right. \\
+ & \left.\mu^{2} \tilde{\mathcal{F}}_{2 ; h}\left(\frac{\Omega}{\mu}\right) \tilde{\mathcal{T}}_{A ; h}^{(+1) *}\left(\frac{\Omega-\Omega_{-}}{\mu}, \frac{\Omega_{+}}{\mu}\right) \tilde{\mathcal{T}}_{A ; h}^{(0)}\left(\Omega_{-}, \Omega\right)+\mu \tilde{\mathcal{F}}_{2 ; h}\left(\frac{\Omega}{\mu^{2}}\right) \tilde{\mathcal{T}}_{A ; h}^{(-1) *}\left(\frac{\Omega_{+}}{\mu}, \frac{\Omega}{\mu}\right) \tilde{\mathcal{T}}_{A ; h}^{(0)}\left(\Omega_{-}, \Omega-\Omega_{+}\right)\right\}, \\
& W_{c ; h}\left(\Omega_{-}, \Omega_{+}\right)=\int \frac{d \Omega}{2 \pi}\left\{\frac{1}{\mu^{3}} \tilde{\mathcal{F}}_{2 ; h}(\Omega) \tilde{\mathcal{T}}_{A ; h}^{(-1)}\left(\Omega \mu, \Omega_{+} \mu\right) \tilde{\mathcal{T}}_{A ; h}^{(-1)}\left(\Omega, \Omega_{+}\right)\right. \\
+ & \left.\frac{1}{\mu^{2}} \tilde{\mathcal{F}}_{2 ; h}(\Omega \mu) \tilde{\mathcal{T}}_{A ; h}^{(-1)}\left[\Omega_{-},\left(\Omega_{+}-\Omega\right) \mu\right] \tilde{\mathcal{T}}_{A ; h}^{(0)}\left(\Omega, \Omega_{+}\right)+\frac{1}{\mu} \tilde{\mathcal{F}}_{2 ; h}\left(\Omega \mu^{2}\right) \tilde{\mathcal{T}}_{A ; h}^{(+1)}\left(\Omega \mu, \Omega_{-} \mu\right) \tilde{\mathcal{T}}_{A ; h}^{(-1)}\left(\Omega+\Omega_{-}, \Omega_{+}\right)\right\} .
\end{aligned}
$$


[1] R. H. Kraichnan, J. Fluid Mech. 41189 (1970).

[2] V.S. L'vov, and I. Procaccia. Computing the Scaling Exponents in Fluid Turbulence from First Principles: the Formal Setup, Physica A, 257 , 0000 (1998). Also \# chao-dyn/9707015.

[3] V.I. Belinicher, V.S. L'vov, and I. Procaccia. Physica A, 254, 215-230 (1998).

[4] U. Frisch. Turbulence: The Legacy of A.N. Kolmogorov (Cambridge University Press, Cambridge, 1995).

[5] V. I. Belinicher, V.S. L'vov, A. Pomyalov and I. Procaccia, submitted in Jour. Stat. Phys. (1998).

[6] V.S. L'vov, E. Podivilov, A. Pomyalov, I. Procaccia and
D. Vandembrouq, Phys. Rev. E, 581811 (1998).

[7] R. H. Kraichnan, J. Math. Phys. 2, 124 (1961).

[8] D. Pierotti, Europhys. Lett. 37, 323 (1997).

[9] G. L. Eyink, Random Coupling Model and SelfConsistent $\epsilon$-Expansion Method, (unpublished).

[10] R. H. Kraichnan, Phys. Fluids 8, 575 (1965).

[11] V.I. Belinicher and V.S. L'vov, Zh. Eksp. Teor. Fiz., 93 1269 (1987), [Soviet Physics - JETP, 66303 (1987)].

[12] G. Parisi and U. Frisch, in Turbulence and Predicatability in Geophysical Fluid Dynamics and Climate Dynamics, eds. M. Ghil, R. Benzi and G. Parisi (North Holland, Amsterdam, 1985) p. 84; T.C. Halsey, M.H. Jensen, L.P. Kadanoff, I. Procaccia and B.I. Shraiman, Phys. Rev A 33, 1141 (1986).

[13] S. Wolfram, The Mathematica book, III edition, (Cambridge Univ. Press, 1998) 